Review

\title{
Copula-based multivariate input modeling
}

\author{
Bahar Biller ${ }^{\mathrm{a}, *}$, Canan Gunes Corlu ${ }^{\mathrm{b}}$ \\ ${ }^{a}$ Tepper School of Business, Carnegie Mellon University, United States \\ ${ }^{\mathrm{b}}$ Department of Industrial Engineering, Bilkent University, Turkey
}

\section{A R T I C L E I N F O}

\section{Article history:}

Received 7 April 2010

Received in revised form

13 February 2012

Accepted 3 April 2012

\begin{abstract}
A B S T R A C T
In this survey, we review the copula-based input models that are well suited to provide multivariate input-modeling support for stochastic simulations with dependent inputs. Specifically, we consider the situation in which the dependence between pairs of simulation input random variables is measured by tail dependence (i.e., the amount of dependence in the tails of a bivariate distribution) and review the techniques to construct copula-based input models representing positive tail dependencies. We complement the review with the parameter estimation from multivariate input data and the randomvector generation from the estimated input model with the purpose of driving the simulation.
\end{abstract}

(c) 2012 Elsevier Ltd. All rights reserved.

\section{Contents}

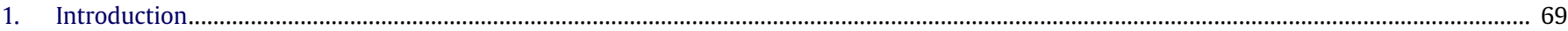

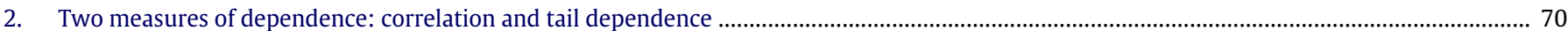

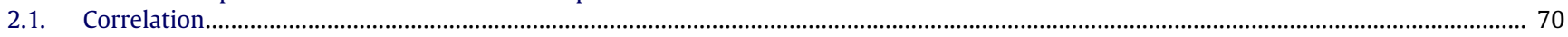

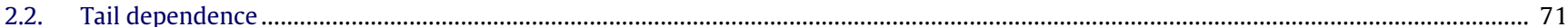

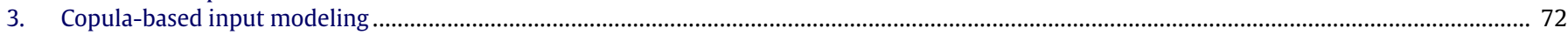

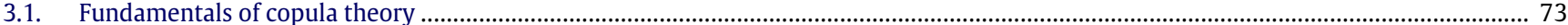

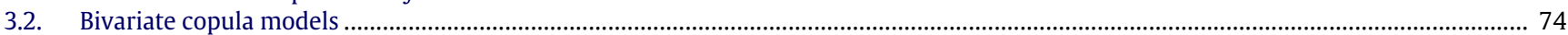

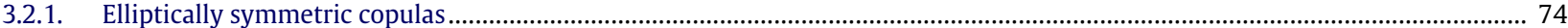

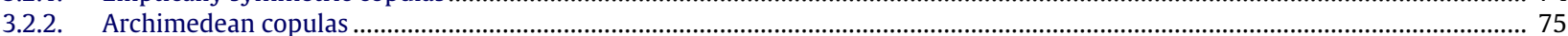

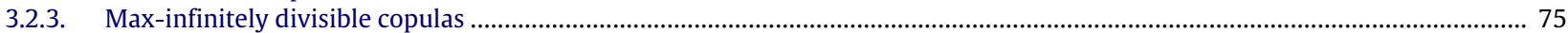

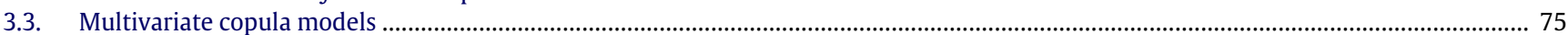

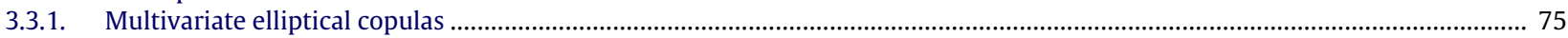

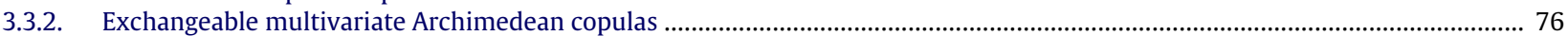

3.3.3. Mixtures of max-infinitely divisible copulas ....................................................................................................................... 76

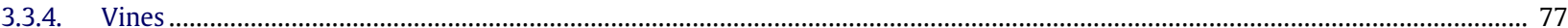

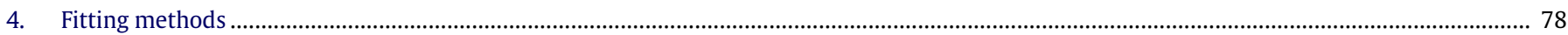

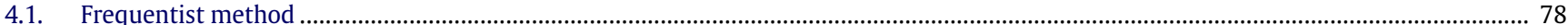

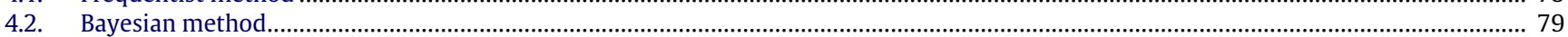

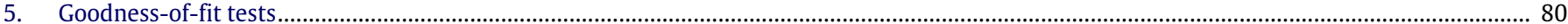

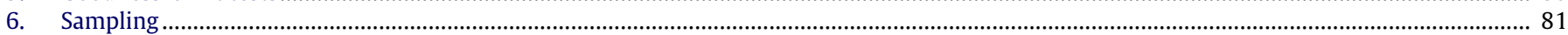

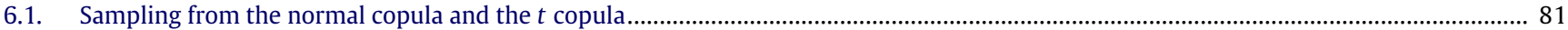

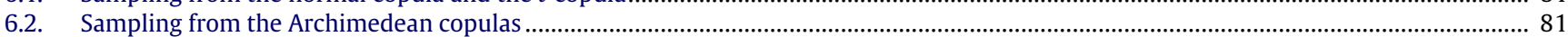

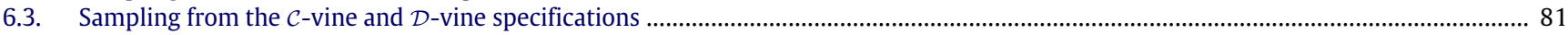

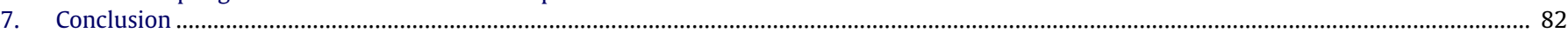

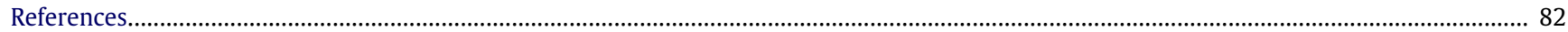

\section{Introduction}

An important step in the design of a stochastic simulation is input modeling; i.e., choosing a probability distribution to

\footnotetext{
* Corresponding author.

E-mail address: billerb@andrew.cmu.edu (B. Biller).
}

represent the inputs of the system being studied. Input modeling is easily performed when the system inputs can be represented as a sequence of independent and identically distributed random variables. Reviews of such input models are available in [1-3].

The focus of this survey is on stochastic simulations with dependent inputs that require the use of flexible multivariate input models to capture their joint distributional properties. Examples 
of dependent inputs in need of multivariate input-modeling support include the processing times of workpieces across work centers [4], the inter-arrival times of file accesses in computer systems [5-7], the medical characteristics of organ-transplant donors and recipients [8], arrival and service processes of Web servers [9], the inter-arrival times of customers in call centers [10], and the product demands and exchange rates of global supply chains [11]. Choosing correlation as the dependence measure, Ghosh and Squillante [9] show that ignoring the correlation between the inter-arrival and service times of Web server queues leads to a $25 \%$ overestimation of the waiting times, while assuming independent and identically distributed inter-arrival times leads to the underestimation of the expected waiting times by a factor of four. Clearly, the independence assumption can lead to very poor estimates of the performance measures when there is actually correlation present, and the consequences of ignoring correlation can be severe. A comprehensive review of the multivariate input models measuring dependence by correlation is available in [12]. Patton [13], on the other hand, demonstrates the need for an input model that captures not only the correlation but also the dependence in the tails of the exchange rate processes. What distinguishes our survey from others is its focus on this measure of dependence, which is known as the tail dependence and defined as the amount of dependence in the tails of a joint distribution. Other applications with focus on tail dependence include Corbett and Rajaram [14], Wagner et al. [15], and Tehrani et al. [16], and they demonstrate that it is imperative to develop multivariate input models that can capture stochastic tail dependencies among the input random variables of stochastic systems.

A close look at the existing literature reveals that multivariate input models can be classified into two types: random vectors and multivariate time series. Specifically, a random vector $\mathbf{X}=\left(X_{1}\right.$, $\left.X_{2}, \ldots, X_{k}\right)^{\prime}$ denotes a collection of $k$ random components, each of which is a real-valued random variable, and it is described by its joint distribution function. A $k$-dimensional time series $\mathbf{X}_{t}=$ $\left\{\left(X_{1, t}, X_{2, t}, \ldots, X_{k, t}\right)^{\prime} ; t=1,2, \ldots\right\}$, on the other hand, denotes a sequence of random vectors observed at times $t=1,2, \ldots$ In this survey, we focus on random vectors and refer the reader to Biller and Ghosh [12] for a comprehensive review of the time-series processes for stochastic simulations. More specifically, we consider multi-dimensional copula-based input models that have the ability to capture a wide variety of dependence structures by describing dependence in a more general manner than correlation. The use of correlation as the measure of dependence in the simulation inputmodeling research has been justified by the fact that making it possible for simulation users to incorporate dependence via correlation, while limited, is substantially better than the practice of ignoring dependence. However, when the simulation inputs are not jointly elliptically distributed and require the use of a joint probability distribution with positive dependence in the tails, correlation is no longer sufficient to describe the dependence structure of these simulation inputs. Therefore, in this survey we go beyond the use of correlation as a dependence measure and present the application of copula theory to multivariate input modeling with the purpose of constructing flexible density models that represent a wide variety of dependence structures. Additionally, we consider the problems of estimating the parameters of the copula-based input models from multivariate data and generating random vectors with the pre-specified marginal distributions and dependence structures to drive stochastic simulations. We refer the reader to Craney and White [17] for input modeling techniques when no data are available.

We organize the remainder of the paper as follows. In Section 2, we introduce correlation and tail dependence as the two measures of dependence that are used for multivariate input modeling with focus on the limitations of correlation as the dependence measure.
In Section 3, we present copula-based input models with the ability to measure tail dependence; Section 3.1 reviews copula theory, Section 3.2 focuses on two-dimensional input models, and Section 3.3 extends the discussion to multivariate input models with three or more component random variables. In Section 4 we describe how to estimate the parameters of the copula-based input models from multivariate data via automated algorithms. We present the goodness-of-fit tests specifically designed for copula-based input models in Section 5. In Section 6, we provide the sampling algorithms that generate random vectors from the copula-based input models quickly and accurately to drive stochastic simulations. We conclude our review with a discussion of promising research areas in Section 7.

\section{Two measures of dependence: correlation and tail depen- dence}

Dependent random vectors are often specified partially in terms of the marginal distributions of their component random variables and pair-wise measures of dependence summarizing how these components interact with each other. Although this may not uniquely or even correctly specify the joint distribution of the random vector, the hope is to find a useful specification for the dependence structure among the components, while sparing the simulation practitioner the task of trying to estimate the full joint distribution. In Section 2.1, we review productmoment correlation and rank correlation as the dependence measures that are often used for this purpose in simulation input modeling. In Section 2.2, we introduce tail dependence and motivate its consideration in this survey as a measure of the dependence captured by neither product-moment correlation nor rank correlation. The dependence measures we consider in each of these sections are pair-wise measures, in that they are used to quantify the dependence between the pairs of random variables. It is important to note that, despite the focus of this survey, correlation and tail dependence are not the only means to measure dependence; we refer the reader to Nelsen [18] for a discussion of alternative measures.

\subsection{Correlation}

The product-moment correlation and the rank correlation are the two widely used measures of dependence in applications of stochastic simulations. Specifically, the rank correlation $r(i, j)$ between random variables $X_{i}$ and $X_{j}$ is defined by

$r(i, j)=\frac{\mathrm{E}\left(F_{i}\left(X_{i}\right) F_{j}\left(X_{j}\right)\right)-\mathrm{E}\left(F_{i}\left(X_{i}\right)\right) \mathrm{E}\left(F_{j}\left(X_{j}\right)\right)}{\sqrt{\operatorname{Var}\left(F_{i}\left(X_{i}\right)\right) \operatorname{Var}\left(F_{j}\left(X_{j}\right)\right)}}$,

where $F_{i}$ is the cumulative distribution function (cdf) of $X_{i}$ [19]. The product-moment correlation $\rho_{\mathbf{X}}(i, j)$ between $X_{i}$ and $X_{j}$ with finite variances $\operatorname{Var}\left(X_{i}\right)$ and $\operatorname{Var}\left(X_{j}\right)$ is, on the other hand, given by

$\rho_{\mathbf{X}}(i, j)=\frac{\operatorname{Cov}\left(X_{i}, X_{j}\right)}{\sqrt{\operatorname{Var}\left(X_{i}\right) \operatorname{Var}\left(X_{j}\right)}}=\frac{\mathrm{E}\left(X_{i} X_{j}\right)-\mathrm{E}\left(X_{i}\right) \mathrm{E}\left(X_{j}\right)}{\sqrt{\operatorname{Var}\left(X_{i}\right) \operatorname{Var}\left(X_{j}\right)}}$,

where $\operatorname{Cov}\left(X_{i}, X_{j}\right)$ is the product-moment covariance between $X_{i}$ and $X_{j}$ [20]. Thus, a correlation of 1 is the maximum possible for bivariate normal random variables $\Phi^{-1}\left(F_{i}\left(X_{i}\right)\right)\left(\equiv Z_{i}\right)$ and $\Phi^{-1}\left(F_{j}\left(X_{j}\right)\right)\left(\equiv Z_{j}\right)$, where $\Phi$ is the cdf of a standard normal random variable. Therefore, taking $\operatorname{Cov}\left(Z_{i}, Z_{j}\right)=1$ is equivalent (in distribution) to setting $Z_{i} \leftarrow \Phi^{-1}(U)$ and $Z_{j} \leftarrow \Phi^{-1}(U)$, where $U$ is a uniform random variable in the interval $(0,1)$ [21]. This definition of $Z_{i}$ and $Z_{j}$ implies that $X_{i} \leftarrow F_{i}^{-1}(U)$ and $X_{j} \leftarrow F_{j}^{-1}(U)$, 

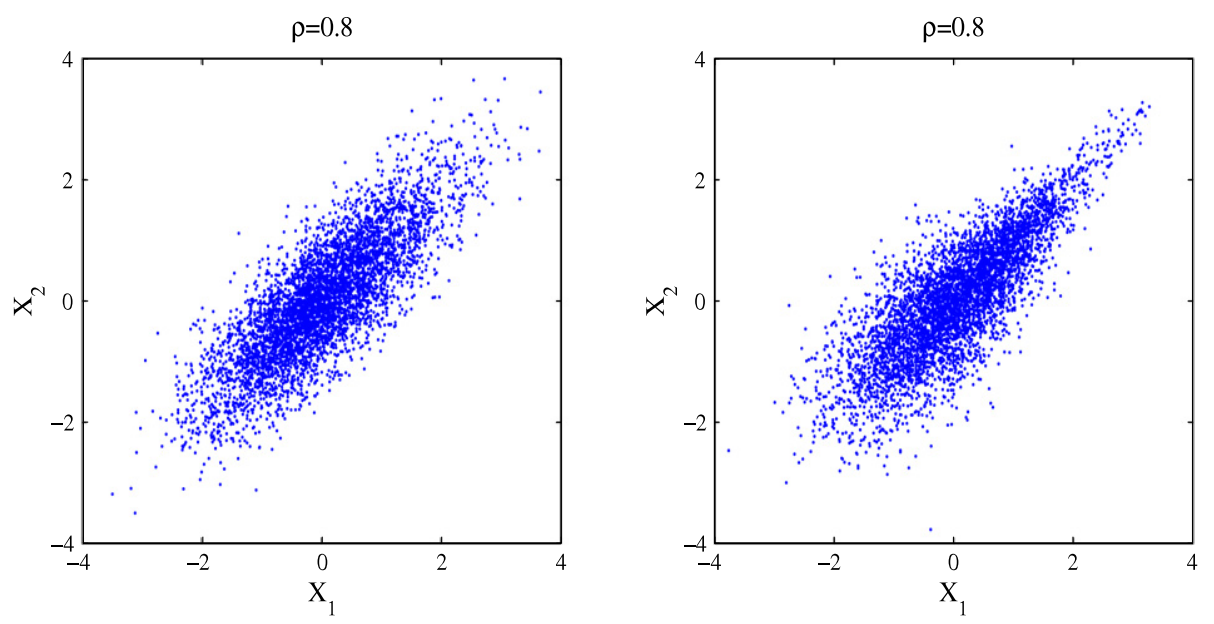

Fig. 1. Input models with standard normal marginals, but with different dependence structures.

from which it follows that $\rho_{\mathbf{X}}(i, j)$ takes on its maximum magnitude. Similarly, taking $\operatorname{Cov}\left(Z_{i}, Z_{j}\right)=-1$ is equivalent (in distribution) to setting $X_{i} \leftarrow F_{i}^{-1}(U)$ and $X_{j} \leftarrow F_{j}^{-1}(1-U)$, in which case the correlation $\rho_{\mathbf{X}}(i, j)$ assumes the minimum possible value for the random variables $X_{i}$ and $X_{j}$. Furthermore, in the special case of jointly normal input random variables, the product-moment correlation $\rho_{\mathbf{X}}(i, j)$ relates to the rank correlation $r(i, j)$ by $\rho_{\mathbf{X}}(i, j)=$ $2 \sin (\pi r(i, j) / 6)[22]$.

Despite its wide use, the product-moment correlation suffers from several limitations that have motivated simulation practitioners to look for alternative measures of dependence (e.g., $[23,12])$ :

(1) The product-moment correlation cannot capture the nonlinear dependence between random variables. Consequently, it fails to model the non-zero dependence in the tails of a bivariate distribution. As an example, Fig. 1 shows 10000 bivariate realizations sampled from two different input models constructed for the random vector $\mathbf{X}=\left(X_{i}, X_{j}\right)^{\prime}$. In both of these models, $X_{i}$ and $X_{j}$ have standard normal marginal distributions and the product-moment correlation $\rho_{\mathbf{X}}(i, j)$ is 0.8 , but with different dependence structures: The first model has the bivariate normal distribution, while the second model has the Gumbel distribution with parameter $\theta$ that takes the value of 3.8 (Section 3.2). More specifically, the dependence in the tails of the joint distribution is zero in the first model, while extreme positive realizations have a tendency to occur together in the second model. Thus, the structure of dependence in the two models cannot be distinguished on the grounds of product-moment correlation alone.

(2) A product-moment correlation of zero between two random variables does not guarantee their independence. For example, the correlation $\rho_{\mathbf{X}}(i, j)$ is zero between $X_{i}$ and $X_{j}$ that are uniformly distributed on the unit circle, but $X_{i}$ and $X_{j}$ are dependent as $X_{i}^{2}+X_{j}^{2}=1$.

(3) A weak product-moment correlation does not imply low dependence. For example, the minimum product-moment correlation between two lognormal random variables with zero means and standard deviations of 1 and $\sigma^{2}$ is the correlation between $e^{Z}$ and $e^{-\sigma Z}$, while the maximum product-moment correlation between these two random variables is the correlation between $e^{Z}$ and $e^{\sigma Z}$, where $Z$ is a standard normal random variable [23]. Although both of these correlations tend to zero with increasing values of $\sigma$, they are highly dependent.
(4) It follows from the definition of the product-moment correlation that $\rho_{\mathbf{X}}(i, j)$ takes values between -1 and 1 , but the actual values $\rho_{\mathbf{X}}(i, j)$ can assume depends on the marginal distributions of the input random variables $X_{i}$ and $X_{j}$ [24]. For example, the attainable interval for the product-moment correlation of two lognormal random variables with zero means and standard deviations of 1 and 2 is [ $-0.090,0.666]$; i.e., it is not possible to find a bivariate distribution with these marginals and a product-moment correlation of 0.7 .

(5) Product-moment correlation is not invariant under transformations of the input random variables. For example, the product-moment correlation between $\log \left(X_{i}\right)$ and $\log \left(X_{j}\right)$ is not the same as the product-moment correlation between $X_{i}$ and $X_{j}$ unless they are independent.

(6) Product-moment correlation is only defined when the variances of the random variables are finite. Therefore, it is not an appropriate dependence measure for heavy-tailed inputs with infinite variances.

The use of the rank correlation as the dependence measure avoids the theoretical deficiencies in 3, 4, 5, and 6. It further provides a natural way to separate the characterization of the component distribution functions $F_{i}\left(X_{i}\right)$ and $F_{j}\left(X_{j}\right)$ from that of the correlation between $X_{i}$ and $X_{j}$. Danaher and Smith [25] use the rank correlation to study the interaction between the length of customer visit to an online store and the purchase amount. A bivariate plot of the visit duration of a customer against the total amount spent by this customer shows that the marginal distributions are far from being normal and the product-moment correlation between the visit duration and the purchase amount is 0.08 , indicating a weak relationship. However, Danaher and Smith compute a stronger dependence via rank correlation with a value of 0.26 . A comprehensive review of similar monotone and transformation-invariant measures of dependence like rank correlation can be found in [18]. However, as in the case of product-moment correlation, the dependence structures of the input models in Fig. 1 cannot be distinguished on the grounds of rank correlation alone, and deficiencies in 1 and 2 remain.

\subsection{Tail dependence}

Motivated by the pitfalls of correlation, focus on the recent multivariate input-modeling research has been finding alternative ways to understand and model dependence by moving away from simple measures of dependence. An alternative measure of dependence, which has been of interest in recent years, is tail dependence; i.e., the amount of dependence in the tails of a joint 

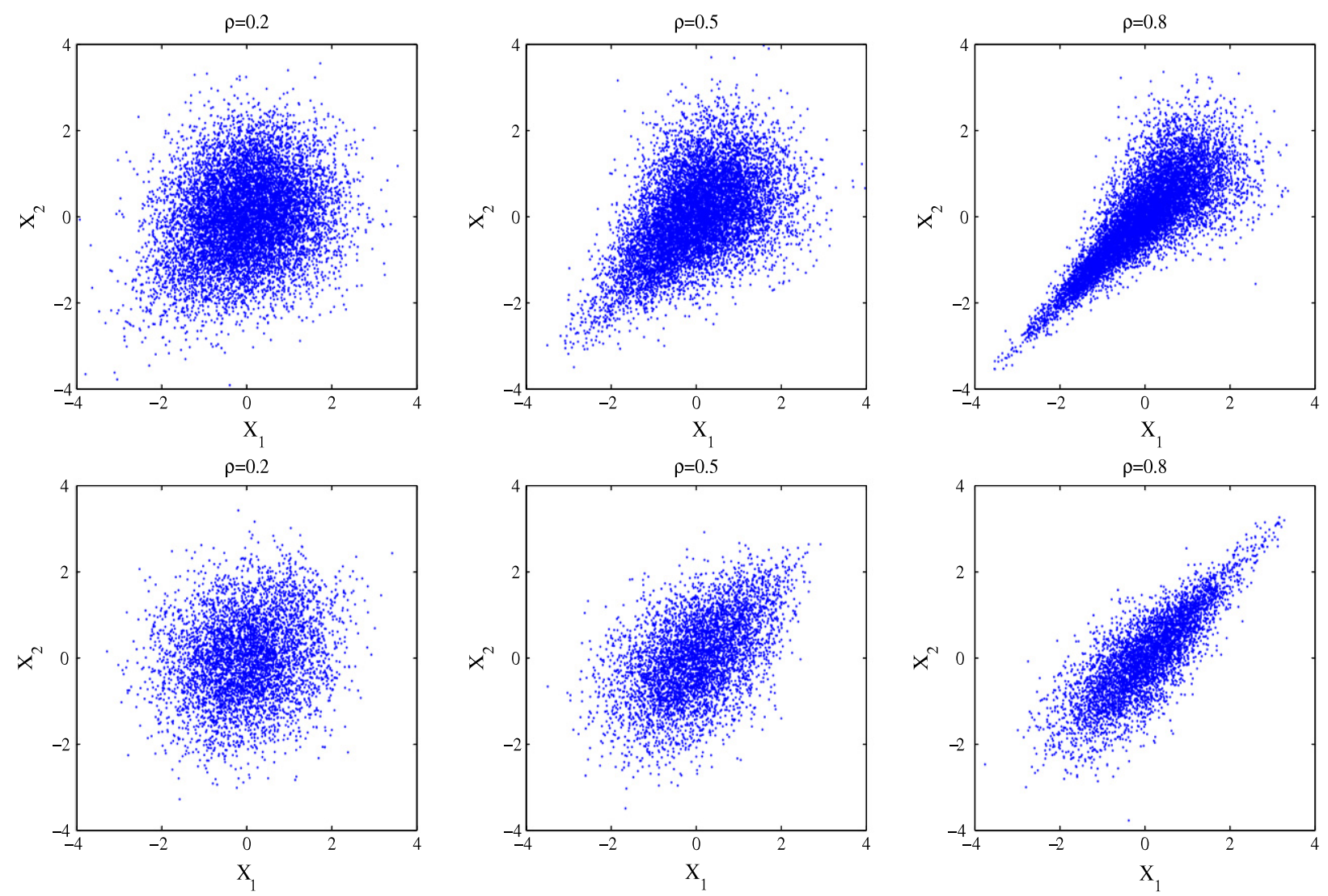

Fig. 2. Examples of bivariate input models with standard normal marginals and positive tail dependencies.

distribution [26]. Specifically, the positive lower-tail dependence $v_{L}(i, j)$ between random variables $X_{i}$ and $X_{j}$ is the amount of dependence in the lower-quadrant tail of the joint distribution of $X_{i}$ and $X_{j}$ and thus, it is given by $\lim _{\ell \downarrow 0} \operatorname{Pr}\left(X_{i} \leq F_{i}^{-1}(\ell) \mid X_{j} \leq\right.$ $\left.F_{j}^{-1}(\ell)\right)$. The positive upper-tail dependence $v_{U}(i, j)$ is, on the other hand, the amount of dependence in the upper-quadrant tail of the joint distribution of $X_{i}$ and $X_{j}$ and thus, it is given by $\lim _{\ell \uparrow 1} \operatorname{Pr}\left(X_{i} \geq F_{i}^{-1}(\ell) \mid X_{j} \geq F_{j}^{-1}(\ell)\right)$. Fig. 2 provides examples of dependence structures for bivariate input models with standard normal marginals, but different positive tail dependencies [27]. The plots of the first, second, and third columns of this illustration are obtained for product-moment correlations of $0.2,0.5$, and 0.8 , respectively. However, the first-row (second-row) plots exhibit greater dependence in the joint lower (upper) tail than in the joint upper (lower) tail; i.e., $v_{L}(i, j)>0$ and $v_{U}(i, j)=0\left(v_{U}(i, j)>0\right.$ and $\left.v_{L}(i, j)=0\right)$. More specifically, the first-row (second-row) plots are obtained from a Clayton (Gumbel) distribution with parameters 0.43 (1.18) for the first column, 1.00 (1.71) for the second column, and 3.11 (3.80) for the third column (Section 2). The plots of Fig. 2 also coincide with the plots of Burr-ParetoLogistic family proposed by Cook and Johnson $[28,29]$. However, most multivariate input models, including the multivariate normal distribution [30], multivariate Johnson translation system [31], and the Normal-To-Anything (NORTA) distribution [32], measure the dependence between simulation inputs using correlation; therefore, they fail to capture the types of dependence structures illustrated in Fig. 2.

The need for input models with asymmetric dependence structures arises in situations where extreme positive realizations have a tendency to occur together. For example, Fortin and Kuzmics [33] show that the stock-return pairs of financial markets exhibit high dependence in the lower tail as well as low dependence in the upper tail of their joint distribution. Similar empirical evidence for the need to measure tail dependence is provided in [34-36]. Patton [13], on the other hand, studies the dependence between mark-dollar and Yen-dollar exchange rates and shows that they are more dependent when they are depreciating than when they are appreciating. Thus, the asymmetric dependence structure of these exchange-rate processes cannot be adequately modeled by the widely used multivariate input models of the simulation inputmodeling literature.

Despite our focus on copula-based input models representing positive tail dependencies, it is important to note that the notion of negative tail dependence has been introduced by Zhang [37]. Specifically, the negative upper-tail dependence, which is called the lower-upper tail dependence in [37], is defined by $\lim _{\ell \downarrow 0} \operatorname{Pr}\left(X_{i} \leq F_{i}^{-1}(\ell) \mid X_{j} \geq F_{j}^{-1}(\ell)\right)$, while the negative lowertail dependence, which is also known as the upper-lower tail dependence, is given by $\lim _{\ell \uparrow 1} \operatorname{Pr}\left(X_{i} \geq F_{i}^{-1}(\ell) \mid X_{j} \leq F_{j}^{-1}(\ell)\right)$. Copula theory reviewed in this paper for positive tail dependence readily extends to these definitions of negative tail dependence. However, most of the available empirical evidence has been for positive tail dependence. Although Sun and Wu [38] provide empirical evidence for the existence of negative tail dependence between the returns of the S\&P 500 index and the returns of the Market Volatility Index, the negative tail dependence is rarely mentioned. Therefore, we restrict the focus of this survey to the representation of positive tail dependence. In the next section, we describe how to utilize copula theory to develop multivariate input models with the ability to capture asymmetric dependence structures, which are characterized by the positive tail dependencies among the simulation input random variables.

\section{Copula-based input modeling}

Copulas have been used extensively for a variety of financial applications including Value-at-Risk calculations [39-41], option 
pricing [42-46], credit risk modeling [47,48], and portfolio optimization $[49,50]$. For a comprehensive review of the applications of copulas in finance, we refer the reader to Patton [51]. However, copulas are not used only for solving financial problems; they are also used for decision and risk analysis [52], aggregation of expert opinions [53], estimation of joint crop yield distributions [54], disruptive event modeling in project management [55], analysis of accident precursors [56,57], and modeling travel behavior by accommodating self-selection effects [58]. Recently, copulas have also been used in marketing to model the purchase behavior of customers [25], and in operations management to model retailer demands [14], supplier defaults [15], and supply disruptions in supply chains [16]. We provide the basics of copula theory in Section 3.1, review bivariate copula models in Section 3.2, and present multivariate copula models with three or more component random variables in Section 3.3.

\subsection{Fundamentals of copula theory}

A way of modeling the dependence among the components of a $k$-dimensional random vector that avoids the pitfalls of correlation is to use a $k$-dimensional copula [18, Definition 2.10.6]:

Definition 1. A $k$-dimensional copula is a function $C_{k}:[0,1]^{k} \rightarrow$ $[0,1]$ with the following properties: (1) For every $\mathbf{u}=\left(u_{1}, u_{2}\right.$, $\left.\ldots, u_{k}\right)$ in $[0,1]^{k}, C_{k}(\mathbf{u})=0$ if at least one coordinate of $\mathbf{u}$ is 0 ; and if all coordinates of $\mathbf{u}$ are 1 except $u_{\ell}$, then $C_{k}(\mathbf{u})=u_{\ell}$ for $\ell=$ $1,2, \ldots, k$. (2) For every $\mathbf{a}=\left(a_{1}, a_{2}, \ldots, a_{k}\right)$ and $\mathbf{b}=\left(b_{1}, b_{2}\right.$, $\left.\ldots, b_{k}\right)$ in $[0,1]^{k}$ such that $\mathbf{a} \leq \mathbf{b}$; i.e., $a_{\ell} \leq b_{\ell}, \ell=1,2, \ldots, k$, and for every $k$-box $[\mathbf{a}, \mathbf{b}]$; i.e., $\left[a_{1}, b_{1}\right] \times\left[\bar{a}_{2}, b_{2}\right] \times \cdots \times\left[a_{k}, b_{k}\right]$, the $C_{k}$-volume given by $\Delta_{\mathbf{a}}^{\mathbf{b}} C_{k}(\mathbf{t})=\Delta_{a_{k}}^{b_{k}} \Delta_{a_{k-1}}^{b_{k-1}} \cdots \Delta_{a_{2}}^{b_{2}} \Delta_{a_{1}}^{b_{1}} C_{k}(\mathbf{t})$ with $\Delta_{a_{\ell}}^{b_{\ell}} C_{k}(\mathbf{t})=C_{k}\left(t_{1}, \ldots, t_{\ell-1}, b_{\ell}, t_{\ell+1}, \ldots, t_{k}\right)-C_{k}\left(t_{1}, \ldots, t_{\ell-1}, a_{\ell}\right.$, $\left.t_{\ell+1}, \ldots, t_{k}\right)$ is $\geq 0$.

The first condition of this definition provides the lower bound on the joint distribution function and insures that the marginal distributions of the copula are uniform. The second condition insures that the probability of observing a point in a $k$-box is nonnegative. Thus, a $k$-dimensional copula is simply a $k$-dimensional distribution function with uniform marginals.

It is important to note that copulas are not the only means to obtain joint distribution functions from uniform marginals. There exist useful families of multivariate uniform distributions that could be the basis for multivariate input modeling: Multivariate Burr distribution [59], multivariate Pareto distribution [60], and multivariate logistic distribution [61] are essentially obtained by transforming uniform marginal distributions to arbitrary marginal distributions. Similarly, Plackett's distribution [62] is obtained by transforming bivariate uniform marginal distributions to arbitrary marginal distributions, while Morgenstern's distribution [63] with uniform marginals is generalized to have arbitrary marginal distributions in [64]. We refer the reader to Chapter 9 and Chapter 10 of Johnson [65] for a detailed discussion on obtaining multivariate distributions with arbitrary marginals from multivariate uniform distributions. This survey, however, focuses on tail dependence and therefore, describes the use of copulas to obtain multivariate distributions with arbitrary marginals and positive tail dependencies.

The use of a copula for representing the joint distribution of a random vector has been studied extensively for the last two decades $[66,67,26,68,18]$. The name "copula" emphasizes the manner in which a $k$-dimensional distribution function is "coupled" to its $k$ (one-dimensional) marginal distributions; this property is formally stated in Sklar's theorem [18, Theorem 2.10.9]:
Theorem 1. Let $F$ be a $k$-dimensional distribution function with marginals $F_{i}, i=1,2, \ldots, k$. Then, there exists a $k$-dimensional copula $C_{k}$ such that for all $x_{i}, i=1,2, \ldots, k$ in domain $\mathfrak{R}^{k}$,

$F\left(x_{1}, x_{2}, \ldots, x_{k}\right)=C_{k}\left(F_{1}\left(x_{1}\right), F_{2}\left(x_{2}\right), \ldots, F_{k}\left(x_{k}\right)\right)$.

If $F_{i}, i=1,2, \ldots, k$ are all continuous, then $C_{k}$ is unique; otherwise, $C_{k}$ is uniquely determined on $\operatorname{Ran} F_{1} \times \operatorname{Ran} F_{2} \times \cdots \times \operatorname{Ran} F_{k}$. Conversely, if $C_{k}$ is a k-dimensional copula and $F_{i}, i=1,2, \ldots, k$ are distribution functions, then the function $F$ is a $k$-dimensional distribution function with marginals $F_{i}, i=1,2, \ldots, k$.

The major implication of this theorem is that copula $C_{k}$ is the joint distribution function of $U_{i} \equiv F_{i}\left(X_{i}\right), i=1,2, \ldots, k$, where the random variables $U_{i}, i=1,2, \ldots, k$ are the probability integral transforms of $X_{i}, i=1,2, \ldots, k$. Thus, each of the random variables $U_{i}, i=1,2, \ldots, k$ follows a uniform distribution in $[0,1]$, regardless of the distributions of the component random variables $X_{i}, i=1,2, \ldots, k$. Moreover, $C_{k}$ can be uniquely determined when the marginal cdfs $F_{i}, i=1,2, \ldots, k$ are all continuous. If the marginal cdfs $F_{i}, i=1,2, \ldots, k$ are all discrete, then $C_{k}$ is uniquely determined on $\operatorname{Ran} F_{1} \times \operatorname{Ran} F_{2} \times \cdots \times \operatorname{Ran} F_{k}$, where $\operatorname{Ran} F_{i}$ is the range of the $\operatorname{cdf} F_{i}$. In any case, the copula $C_{k}$ captures the dependence structure of the joint cdf $F$ and it can be written as $C_{k}\left(u_{1}, u_{2}, \ldots, u_{k}\right)=F\left(F_{1}^{-1}\left(u_{1}\right), F_{2}^{-1}\left(u_{2}\right), \ldots, F_{k}^{-1}\left(u_{k}\right)\right)$, where $F_{i}^{-1}$ is the generalized inverse of the marginal cdf $F_{i}$ [18, Corollary 2.10.10].

The practical premise of Sklar's theorem in multivariate input modeling is that the joint distribution $F$ can be constructed by choosing the marginal distributions $F_{i}, i=1,2, \ldots, k$ and the copula density function $c_{k}$ separately. More specifically, any joint probability density function (pdf) $f$ can be written as a product of its marginal pdfs $f_{i}, i=1,2, \ldots, k$ and copula density function $c_{k}$ for differentiable cdfs $F_{i}, i=1,2, \ldots, k$ and differentiable copula $C_{k}$; i.e.,

$f\left(x_{1}, x_{2}, \ldots, x_{k}\right)=c_{k}\left(F_{1}\left(x_{1}\right), F_{2}\left(x_{2}\right), \ldots, F_{k}\left(x_{k}\right)\right) \cdot \prod_{i=1}^{k} f_{i}\left(x_{i}\right)$.

The marginal pdf $f_{i}$ is obtained from $\partial F_{i}\left(x_{i}\right) / \partial x_{i}$, while $\partial^{k} C_{k}\left(u_{1}\right.$, $\left.u_{2}, \ldots, u_{k}\right) /\left(\partial u_{1} \partial u_{2} \cdots \partial u_{k}\right)$ provides the $k$-dimensional copula density function $c_{k}$, encoding all of the information about the dependencies among the random variables $X_{i}, i=1,2, \ldots, k$. Thus, $c_{k}$ takes the value of 1 when $X_{i}, i=1,2, \ldots, k$ are independent, and the joint density function reduces to the product of only the marginal pdfs.

There are numerous parametric families of copulas proposed in the literature, emphasizing different distributional properties. In this survey, we distinguish these copulas on the grounds of the tail dependence they capture; i.e., some copulas assign the value of zero to the tail dependence, while others represent positive lowertail dependence and/or positive upper-tail dependence. Next, we present a copula-based definition of the tail dependence for a bivariate input model with copula $C_{2}$ [26]:

Definition 2. If a two-dimensional copula $C_{2}$ is such that $\lim _{u \downarrow 0} C_{2}$ $(u, u) / u=v_{L}$ exists, then $C_{2}$ has lower-tail dependence if $v_{L} \in$ $(0,1]$ and no lower-tail dependence if $v_{L}=0$. Similarly, if $\lim _{u \uparrow 1}\left(1-2 u+C_{2}(u, u)\right) /(1-u)=v_{U}$ exists, then $C_{2}$ has upper-tail dependence if $v_{U} \in(0,1]$ and no upper-tail dependence if $v_{U}=0$.

A close look at the existing literature reveals that one of the widely used copulas for bivariate input modeling is the two-dimensional normal distribution. The application of Definition 2 with the two-dimensional copula $C_{2}$ replaced by the two-dimensional standard normal cdf having correlation $\rho(1,2) \in(-1,1)$ between random variables $Z_{1}\left(\equiv \Phi^{-1}\left(F_{1}\left(X_{1}\right)\right)\right)$ and $Z_{2}\left(\equiv \Phi^{-1}\left(F_{2}\left(X_{2}\right)\right)\right)$ results in the computation of zero for both 
Table 1

Bivariate copulas and their tail-dependence functions.

\begin{tabular}{|c|c|c|c|}
\hline Copula & Parameter & $v_{L}$ & $v_{U}$ \\
\hline \multicolumn{4}{|l|}{ Elliptically symmetric } \\
\hline Normal & $-1<\rho<1$ & 0 & 0 \\
\hline$t$ & $-1<\rho<1,0<d<\infty$ & $2 t_{d+1}\left(\sqrt{\frac{(d+1)(1-\rho)}{(1+\rho)}}\right)$ & $2 t_{d+1}\left(\sqrt{\frac{(d+1)(1-\rho)}{(1+\rho)}}\right)$ \\
\hline \multicolumn{4}{|l|}{ Archimedean } \\
\hline Clayton & $\theta \geq 0$ & $2^{-1 / \theta}$ & 0 \\
\hline \multirow[t]{3}{*}{ Gumbel } & $\theta \geq 1$ & 0 & $2-2^{1 / \theta}$ \\
\hline & $\theta \geq 1$ & $2^{-1 / \theta}$ & $2-2^{1 / \theta}$ \\
\hline & $\theta_{1}>0, \theta_{2} \geq 1$ & $2^{-1 /\left(\theta_{1} \theta_{2}\right)}$ & $2-2^{1 / \theta_{2}}$ \\
\hline Max-infinitely divisible & $\theta_{1} \geq 0, \theta_{2} \geq 1$ & $2^{-1 / \theta_{1}}$ & $2-2^{1 / \theta_{2}}$ \\
\hline
\end{tabular}

the lower-tail dependence $\left(v_{L}(1,2)=0\right)$ and the upper-tail dependence $\left(v_{U}(1,2)=0\right)$ between random variables $X_{1}$ and $X_{2}$. Thus, regardless of the correlation $\rho(1,2) \in(-1,1)$ we choose, extreme events appear to occur independently in $X_{1}$ and $X_{2}$ when we go far enough into the tails. This explains why a bivariate input model building on the normal distribution fails to represent positive tail dependencies.

This discussion readily extends to the multi-dimensional setting with $k \geq 2$. A well known multivariate input model building also on the normal distribution is the NORTA random vector introduced by Cario and Nelson [32]. The goal of this input model is to match the pre-specified properties of the random vector $\mathbf{X}=$ $\left(X_{1}, X_{2}, \ldots, X_{k}\right)^{\prime}$, i.e., the marginal distributions $F_{i}, i=1,2, \ldots, k$ and the input correlations $\rho_{\mathbf{X}}(i, j), i, j=1,2, \ldots, k$, so as to drive the simulation with random vectors that have these properties. Therefore, the construction of the NORTA random vector builds on (1) applying the probability integral transform $F_{i}$ to the input random variable $X_{i}$, which results in the uniform random variable $U_{i}$ (i.e., $F_{i}\left(X_{i}\right)=U_{i}$ ); and (2) applying the inverse cdf $\Phi^{-1}$ to $U_{i}$ from which the standard normal random variable $Z_{i}$ is obtained (i.e., $Z_{i}=\Phi^{-1}\left(F_{i}\left(X_{i}\right)\right)$ ). Consequently, we obtain the joint cdf $F$ of the NORTA random vector $\mathbf{X}$ as follows:

$$
\begin{aligned}
F & \left(x_{1}, x_{2}, \ldots, x_{k}\right) \\
& =\operatorname{Pr}\left(X_{i} \leq x_{i} ; i=1,2, \ldots, k\right) \\
& =\operatorname{Pr}\left(F_{i}\left(X_{i}\right) \leq F_{i}\left(x_{i}\right) ; i=1,2, \ldots, k\right) \\
& =\operatorname{Pr}\left(U_{i} \leq u_{i} ; i=1,2, \ldots, k\right) \\
& =\operatorname{Pr}\left(\Phi^{-1}\left(U_{i}\right) \leq \Phi^{-1}\left(u_{i}\right) ; i=1,2, \ldots, k\right) \\
& =\operatorname{Pr}\left(Z_{i} \leq \Phi^{-1}\left(u_{i}\right) ; i=1,2, \ldots, k\right) \\
& =\Phi_{k}\left(\Phi^{-1}\left(u_{1}\right), \Phi^{-1}\left(u_{2}\right), \ldots, \Phi^{-1}\left(u_{k}\right) ; \Sigma_{k}\right) \\
& =\Phi_{k}\left(\Phi^{-1}\left(F_{1}\left(x_{1}\right)\right), \Phi^{-1}\left(F_{2}\left(x_{2}\right)\right), \ldots, \Phi^{-1}\left(F_{k}\left(x_{k}\right)\right) ; \Sigma_{k}\right) .
\end{aligned}
$$

In this representation, $\Phi_{k}\left(\cdot ; \Sigma_{k}\right)$ is the joint cdf of the standard normal random vector $\mathbf{Z}=\left(Z_{1}, Z_{2}, \ldots, Z_{k}\right)^{\prime}$ with the correlation matrix $\Sigma_{k} \equiv[\rho(i, j) ; i, j=1,2, \ldots, k]$, where $\rho(i, j)$ is the correlation between the random variables $Z_{i}$ and $Z_{j}$. The function $\Phi_{k}\left(\cdot ; \Sigma_{k}\right)$, which is simply the $k$-dimensional normal copula, couples the arbitrary marginal cdfs $F_{i}, i=1,2, \ldots, k$ with the correlation matrix $\boldsymbol{\Sigma}_{k}$ to obtain the joint distribution function $F$. Thus, the dependence structure of the $k$-dimensional NORTA distribution is represented by the $k$-dimensional normal copula, explaining the failure of the NORTA distribution to represent non-zero tail dependencies. This result further extends to the multivariate normal distribution and the multivariate Johnson translation system, which are the special cases of the NORTA distribution. Specifically, we obtain the multivariate normal distribution by allowing each of the NORTA components to have a univariate normal distribution; we obtain the multivariate Johnson translation system by letting each component of the NORTA random vector have a univariate Johnson distribution [69].

A solution to the problem of capturing asymmetric dependence structures with positive tail dependencies is to replace the $k$ dimensional normal copula of the NORTA random vector $\mathbf{X}$ with a $k$-dimensional copula having the ability to match the pre-specified values of lower-tail dependencies $v_{L}(i, j), i, j=1,2, \ldots, k$ and upper-tail dependencies $v_{U}(i, j), i, j=1,2, \ldots, k$. Section 3.2 reviews the bivariate copula models that can be used for this purpose along with their tail dependence properties (i.e., $k=2$ ). Focusing on random vectors with three or more components (i.e., $k \geq 3$ ), Section 3.3 considers the representation of tail dependencies by multivariate input models.

\subsection{Bivariate copula models}

The existing literature contains numerous parametric families of bivariate copulas, emphasizing different distributional properties $[26,68,18]$. In this survey, we consider the property of tail dependence. Table 1 provides the bivariate copulas that can be used for capturing this measure of dependence between any pair of random variables.

\subsubsection{Elliptically symmetric copulas}

Both the normal copula and the $t$ copula fall into the class of elliptically symmetric copulas, which are introduced in [70] and discussed comprehensively in [71] as the generalizations of the normal copula to those with elliptically symmetric contours. Thus, the elliptically symmetric copulas inherit many of the tractable properties of the normal copula and maintain the advantage of being easy to sample. Specifically, the bivariate normal copula with the dependence parameter $\rho \in(-1,1)$ is given by

$$
\begin{aligned}
C_{2}\left(u_{1}, u_{2} ; \rho\right)= & \int_{-\infty}^{\Phi^{-1}\left(u_{1}\right)} \int_{-\infty}^{\Phi^{-1}\left(u_{2}\right)} \frac{1}{2 \pi \sqrt{1-\rho^{2}}} \\
& \times \exp \left\{-\frac{z_{1}^{2}-2 \rho z_{1} z_{2}+z_{2}^{2}}{2\left(1-\rho^{2}\right)}\right\} d z_{1} d z_{2},
\end{aligned}
$$

while the bivariate $t$ copula with the degrees of freedom $d \in$ $(0, \infty)$ is given by

$$
\begin{aligned}
C_{2}\left(u_{1}, u_{2} ; \rho, d\right)= & \int_{-\infty}^{t_{d}^{-1}\left(u_{1}\right)} \int_{-\infty}^{t_{d}^{-1}\left(u_{2}\right)} \frac{1}{2 \pi \sqrt{1-\rho^{2}}} \\
& \times\left\{1+\frac{z_{1}^{2}-2 \rho z_{1} z_{2}+z_{2}^{2}}{d\left(1-\rho^{2}\right)}\right\}^{-(d+2) / 2} d z_{1} d z_{2},
\end{aligned}
$$

where the parameter $\rho$ corresponds to a dependence parameter when $d>2$. An important distinction between these two copulas is that the normal copula assigns the value of zero to the tail dependencies, while both the lower-tail dependence and the upper-tail dependence captured by the $t$ copula are given by $2 t_{d+1}(\sqrt{d+1} \sqrt{1-\rho} / \sqrt{1+\rho})$, where $t_{d}$ denotes the univariate $t$ distribution function with $d$ degrees of freedom [23]. Thus, the $t$ copula assumes positive tail dependence even for $\rho=0$, but the symmetry in the dependence structure (i.e., the equivalence between the lower-tail dependence and the upper-tail dependence) restricts its use for bivariate input modeling; i.e., a property that is shared by all elliptically symmetric copulas. 


\subsubsection{Archimedean copulas}

A family of non-elliptical copulas with the ability to capture asymmetric dependence structures (i.e., different values for lowertail and upper-tail dependencies) is the class of Archimedean copulas. These copulas are analytically tractable in the sense that many of their properties can be derived using elementary calculus [66]. Specifically, an Archimedean copula with parameter $\theta$ is of the form $C_{2}\left(u_{1}, u_{2} ; \theta\right)=\phi^{-1}\left(\phi\left(u_{1} ; \theta\right)+\phi\left(u_{2} ; \theta\right) ; \theta\right)$, where $\phi^{-1}(\cdot ; \theta)$ is the pseudo-inverse of $\phi(\cdot ; \theta):[0,1] \rightarrow[0, \infty]$, which is a continuous, strictly decreasing, and convex generator function satisfying $\phi(1 ; \theta)=0$. Different generator functions lead to different types of Archimedean copulas. For instance, the generator $\phi(t ; \theta)=\left(t^{-\theta}-1\right) / \theta$ produces the Clayton copula $C_{2}\left(u_{1}, u_{2} ; \theta\right)=$ $\left(u_{1}^{-\theta}+u_{2}^{-\theta}-1\right)^{-1 / \theta}$ with $0 \leq \theta<\infty$, leading to a lower-tail dependence of $v_{L}(1,2)=2^{-1 / \theta}$ between random variables $X_{1}$ and $X_{2}$ [72]. This is the copula function used for obtaining the first-row plots of Fig. 2, while the second-row plots are obtained from the generator function $\phi(t ; \theta)=(-\log t)^{\theta}$, which leads to the Gumbel copula $C_{2}\left(u_{1}, u_{2} ; \theta\right)=\exp \left(-\left(\left(-\log u_{1}\right)^{\theta}+\left(-\log u_{2}\right)^{\theta}\right)^{1 / \theta}\right)$, $1 \leq \theta<\infty$ with an upper-tail dependence of $v_{U}(1,2)=2-$ $2^{1 / \theta}$ [73]. Thus, neither the Clayton copula nor the Gumbel copula can simultaneously represent positive lower-tail and uppertail dependencies. The generator function defined as $\phi(t ; \theta)=$ $\left(t^{-1}-1\right)^{\theta}$, however, captures both the lower-tail dependence and the upper-tail dependence. More specifically, it produces the copula $C_{2}\left(u_{1}, u_{2} ; \theta\right)=\left(1+\left(\left(u_{1}^{-1}-1\right)^{\theta}+\left(u_{2}^{-1}-1\right)^{\theta}\right)^{1 / \theta}\right)^{-1}$ with $\theta \geq 1$, leading to a lower-tail dependence of $v_{L}(1,2)=2^{-1 / \theta}$ and an upper-tail dependence of $v_{U}(1,2)=2-2^{1 / \theta}$ [18]. However, the values $v_{L}(1,2)$ and $v_{U}(1,2)$ can take are limited by the copula parameter $\theta$.

A way to overcome this limitation of a single-parameter copula is to construct a two-parameter Archimedean copula. This can be done by using a composite generator function of the form $\phi\left(t ; \theta_{1}, \theta_{2}\right)=\left(\phi\left(t^{\theta_{1}}\right)\right)^{\theta_{2}}$. For example, defining the composite generator function $\phi\left(t ; \theta_{1}, \theta_{2}\right)$ as $\left(t^{-\theta_{1}}-1\right)^{\theta_{2}}$ with $\theta_{1}>0$ and $\theta_{2} \geq$ 1 leads to the two-parameter Archimedean copula $C_{2}\left(u_{1}, u_{2} ; \theta\right)=$ $\left(\left(\left(u_{1}^{\theta_{1}}-1\right)^{\theta_{2}}+\left(u_{2}^{\theta_{1}}-1\right)^{\theta_{2}}\right)^{1 / \theta_{1}}+1\right)^{-1 / \theta_{1}}$ with a lower-tail dependence of $v_{L}(1,2)=2^{-1 /\left(\theta_{1} \theta_{2}\right)}$ and an upper-tail dependence of $v_{U}(1,2)=2-2^{1 / \theta_{2}}$. The upper-tail dependence $v_{U}(1,2)$ can take any value between 0 and 1, providing more flexibility than the single-parameter Archimedean copula in modeling the amount of dependence in the upper-quadrant tail of the underlying joint distribution function. However, the value the lower-tail dependence $v_{L}(1,2)$ can assume is restricted by the value of the upper-tail dependence $v_{U}(1,2)$. This modeling challenge is overcome by the max-infinitely divisible copulas introduced in the next section.

\subsubsection{Max-infinitely divisible copulas}

The lower-tail dependence $v_{L}(1,2) \in(0,1]$ and the uppertail dependence $v_{U}(1,2) \in(0,1]$ between random variables $X_{1}$ and $X_{2}$ can be jointly represented by using a family of copulas of the form $C_{2}\left(u_{1}, u_{2}\right)=\Theta\left(-\log K\left(e^{-\Theta^{-1}\left(u_{1}\right)}, e^{-\Theta^{-1}\left(u_{2}\right)}\right)\right)$, where $\Theta$ is a Laplace transformation and $K$ is a max-infinitely divisible bivariate copula between random variables $U_{1}=F_{1}\left(X_{1}\right)$ and $U_{2}=F_{2}\left(X_{2}\right)$. Specifically, the distribution function $K$ is maxinfinitely divisible if $K^{\alpha}$ is a distribution function for every $\alpha>$ 0 [74]. Furthermore, if $K$ is an Archimedean copula, then $C_{2}$ is an Archimedean copula. For example, letting $K$ be the oneparameter Clayton copula with parameter $\theta_{1} \geq 0$ and allowing $\Theta$ to be the Laplace transformation satisfying $\Theta(t)=1-(1-$ $\left.e^{-t}\right)^{1 / \theta_{2}}$ with parameter $\theta_{2} \geq 1$ results in the two-parameter copula of the form $C_{2}\left(u_{1}, u_{2} ; \theta_{1}, \theta_{2}\right)=\phi^{-1}\left(\phi\left(u_{1} ; \theta_{1}, \theta_{2}\right)+\right.$ $\left.\phi\left(u_{2} ; \theta_{1}, \theta_{2}\right) ; \theta_{1}, \theta_{2}\right)$, where $\phi\left(t ; \theta_{1}, \theta_{2}\right)=\left(1-(1-t)^{\theta_{2}}\right)^{-\theta_{1}}-$ 1 [26]. The application of Definition 2 to this copula function results in the lower-tail dependence $v_{L}(1,2)=2^{-1 / \theta_{1}}$ and the uppertail dependence $v_{U}(1,2)=2-2^{1 / \theta_{2}}$. Thus, parameter $\theta_{1}\left(\theta_{2}\right)$ is used only for adjusting the lower-tail (upper-tail) dependence $v_{L}(1,2)\left(v_{U}(1,2)\right)$; i.e., the use of this two-parameter Archimedean copula for bivariate input modeling allows both the lower-tail dependence and the upper-tail dependence to assume arbitrary values in $[0,1]$. Further discussion on two-parameter bivariate copulas can be found in [26].

\subsection{Multivariate copula models}

In this section, we discuss the extension of the bivariate copula models presented in Section 3.2 to three or more random variables. Specifically, Section 3.3.1 reviews the multivariate elliptical copulas; Section 3.3.2 presents the exchangeable multivariate Archimedean copulas; Section 3.3.3 provides the mixtures of max-infinitely divisible copulas; and finally Section 3.3.4 reviews the vine specifications that are known to be the most flexible multivariate copula models with the ability to represent asymmetric dependence structures with positive tail dependencies.

\subsubsection{Multivariate elliptical copulas}

Two widely used copulas for representing the joint distribution of three or more input random variables are the normal copula associated with the multivariate normal distribution and the $t$ copula associated with the multivariate $t$ distribution. Each of these copulas is a member of the elliptical copula family that is particularly easy to use for driving stochastic simulations with multiple inputs. Specifically, the multivariate normal copula with the positive definite correlation matrix $\boldsymbol{\Sigma}_{k} \equiv[\rho(i, j) ; i, j=1,2$, $\ldots, k]$ is given by

$$
\begin{aligned}
C_{k} & \left(u_{1}, u_{2}, \ldots, u_{k} ; \mathbf{\Sigma}_{k}\right) \\
= & \Phi_{k}\left(\Phi^{-1}\left(u_{1}\right), \Phi^{-1}\left(u_{2}\right), \ldots, \Phi^{-1}\left(u_{k}\right) ; \Sigma_{k}\right), \\
= & \int_{-\infty}^{\Phi^{-1}\left(u_{1}\right)} \ldots \int_{-\infty}^{\Phi^{-1}\left(u_{k}\right)} \frac{1}{(2 \pi)^{k / 2}\left|\Sigma_{k}\right|^{1 / 2}} \\
& \quad \times \exp \left(-\frac{1}{2} \mathbf{z}^{\prime} \Sigma_{k}^{-1} \mathbf{z}\right) d z_{1} \ldots d z_{k},
\end{aligned}
$$

where $\mathbf{z}$ denotes the vector $\left(z_{1}, z_{2}, \ldots, z_{k}\right)^{\prime}$. The limitation of this multivariate copula in representing positive tail dependence becomes apparent in modeling investor's default risk $[75,76]$. For further discussion on the limitations of the multivariate normal copula, we refer the reader to Lipton and Rennie [77], Donnelly and Embrechts [78] and Brigo et al. [79].

The multivariate $t$ copula with the parameters $\boldsymbol{\Sigma}_{k} \equiv[\rho(i, j)$; $i, j=1,2, \ldots, k]$ and $d \in(0, \infty)$ is given by

$$
\begin{aligned}
C_{k}\left(u_{1}, u_{2}, \ldots, u_{k} ; \Sigma_{k}, d\right) & t\left(t_{d}^{-1}\left(u_{1}\right), t_{d}^{-1}\left(u_{2}\right), \ldots, t_{d}^{-1}\left(u_{k}\right) ; \Sigma_{k}, d\right), \\
= & \int_{-\infty}^{t_{d}^{-1}\left(u_{1}\right)} \cdots \int_{-\infty}^{t_{d}^{-1}\left(u_{k}\right)} \frac{\Gamma\left(\frac{d+k}{2}\right)\left|\Sigma_{k}\right|^{-1 / 2}}{\Gamma\left(\frac{d}{2}\right)(d \pi)^{k / 2}} \\
& \times\left(1+\frac{1}{d} \mathbf{z}^{\prime} \Sigma_{k}^{-1} \mathbf{z}\right)^{-\frac{d+k}{2}} d z_{1} \ldots d z_{k} .
\end{aligned}
$$

Even for $\rho(i, j)=0$, the multivariate $t$ copula represents symmetric tail dependencies $v_{L}(i, j)=v_{U}(i, j)=2 t_{d+1}(-\sqrt{d+1}$ $\sqrt{1-\rho(i, j)} / \sqrt{1+\rho(i, j)})$, as can be deduced from Table 1 by focusing on the interaction between random variables $X_{i}$ and $X_{j}$. Thus, the multivariate $t$ copula fails to capture asymmetric dependence structures with different values for lower-tail and uppertail dependencies. In the following section, this limitation of the multivariate $t$ copula is overcome by using the exchangeable Archimedean copula for multivariate input modeling. 
We conclude this section by noting that the class of the elliptically symmetric copulas includes the logistic copula [71], the exponential power copula [80], and the generalized $t$ copula $[81,82]$. However, not all generalized $t$ copulas are elliptically symmetric; they allow for different degrees of freedom and different types of dependencies among the components of the random vector. We refer the reader to Mendes and Arslan [82] for the characterization of the tail dependencies that can be captured by the generalized $t$ copulas.

\subsubsection{Exchangeable multivariate Archimedean copulas}

The extension of the Archimedean copula introduced in Section 3.2 for bivariate input modeling is the $k$-dimensional Archimedean copula of the following form:

$$
\begin{aligned}
& C_{k}\left(u_{1}, u_{2}, \ldots, u_{k} ; \theta\right) \\
& \quad=\phi^{-1}\left(\phi\left(u_{1} ; \theta\right)+\phi\left(u_{2} ; \theta\right)+\cdots+\phi\left(u_{k} ; \theta\right) ; \theta\right) .
\end{aligned}
$$

$\phi(\cdot ; \theta):[0,1] \rightarrow[0, \infty)$ is a continuous, strictly decreasing function that satisfies $\phi(0 ; \theta)=\infty$ and $\phi(1 ; \theta)=0$. Additionally, $\phi^{-1}(\cdot ; \theta)$ is a completely monotonic function on $[0, \infty)$ (i.e., $(-1)^{\ell} \partial^{\ell} \phi^{-1}(t ; \theta) / \partial t^{\ell} \geq 0$ for all $t \in(0, \infty)$ and $\ell=0,1$, $\ldots, \infty)[83]$. This is a necessary and sufficient condition for the function $C_{k}(\cdot ; \theta)$ to define a copula, and this condition is satisfied by both the $k$-dimensional Clayton copula and the $k$-dimensional Gumbel copula.

Specifically, the pseudo-inverse of the generator function of the Clayton copula; i.e., $\phi^{-1}(t ; \theta)=(1+t)^{-1 / \theta}$ is completely monotonic on $[0, \infty)$. Therefore, the $k$-dimensional Clayton copula with $\theta>0$ is given by

$$
C_{k}\left(u_{1}, u_{2}, \ldots, u_{k} ; \theta\right)=\left(u_{1}^{-\theta}+u_{2}^{-\theta}+\cdots+u_{k}^{-\theta}-k+1\right)^{-1 / \theta} \text {. }
$$

Due to its ability to represent the joint probability of component random variables taking very small values together, Tehrani et al. [16] use this copula function to model dependent disruptions in supply chains caused by catastrophic events. Wagner et al. [15], on the other hand, use the multivariate Clayton copula for investigating the impact of positive default dependencies on the design of supplier portfolios. However, the $k$-dimensional Clayton copula assumes a lower-tail dependence of $2^{-1 / \theta}$ and an uppertail dependence of zero between all pairs of its components (i.e., $v_{L}(i, j)=2^{-1 / \theta}$ and $v_{U}(i, j)=0$ for $i=1,2, \ldots, k$ and $j=i, i+1$, $\ldots, k)$, limiting its ability to perform flexible dependence modeling.

Similarly, the pseudo-inverse of the generator function of the Gumbel copula; i.e., $\phi^{-1}(t ; \theta)=\exp \left(-t^{1 / \theta}\right)$ is completely monotonic on $[0, \infty)$. Thus, the two-dimensional Gumbel copula of Section 3.2 is generalized to the following $k$-dimensional copula with $\theta \geq 1$ :

$$
\begin{aligned}
& C_{k}\left(u_{1}, u_{2}, \ldots, u_{k} ; \theta\right) \\
& \quad=\exp \left(-\left(\left(-\log u_{1}\right)^{\theta}+\left(-\log u_{2}\right)^{\theta}+\cdots+\left(-\log u_{k}\right)^{\theta}\right)^{1 / \theta}\right) .
\end{aligned}
$$

The functional form of this copula leads to the tail dependencies of $v_{L}(i, j)=0$ and $v_{U}(i, j)=2-2^{1 / \theta}$ for $i=1,2, \ldots, k$ and $j=i$, $i+1, \ldots, k$.

The bivariate two-parameter Archimedean copulas can also be generalized to be the $k$-dimensional Archimedean copulas. As an example, we consider the composite generator function $\phi\left(t ; \theta_{1}, \theta_{2}\right)=\left(t^{-\theta_{1}}-1\right)^{\theta_{2}}$ with $\theta_{1}>0$ and $\theta_{2} \geq 1$. Since the pseudo-inverse of this generator function is completely monotonic on $[0, \infty)$, we obtain the following $k$-dimensional copula:

$$
\begin{aligned}
& C_{k}\left(u_{1}, u_{2}, \ldots, u_{k} ; \theta_{1}, \theta_{2}\right) \\
& \quad=\left(\left(\left(u_{1}^{-\theta_{1}}-1\right)^{\theta_{2}}+\left(u_{2}^{-\theta_{1}}-1\right)^{\theta_{2}}+\cdots+\left(u_{k}^{-\theta_{1}}-1\right)^{\theta_{2}}\right)^{1 / \theta_{2}}+1\right)^{-1 / \theta_{1}} .
\end{aligned}
$$

Therefore, the tail dependencies $v_{L}(i, j)$ and $v_{U}(i, j)$ are, respectively, identified as $2^{-1 /\left(\theta_{1} \theta_{2}\right)}$ and $2-2^{1 / \theta_{2}}$ for $i=1,2, \ldots, k$ and $j=i, i+1, \ldots, k$.

\subsubsection{Mixtures of max-infinitely divisible copulas}

A flexible bivariate copula with the ability to represent any pair of lower-tail and upper-tail dependencies is the max-infinitely divisible bivariate copula (Section 3.2). Thus, a natural question to ask is whether it is possible to build on the mixtures of max-infinitely divisible bivariate copulas so as to construct a $k$-dimensional copula that would match arbitrary values of $v_{L}(i, j)>0$ and $v_{U}(i, j)>0$ for $i=1,2, \ldots, k$ and $j=i, i+1$, $\ldots, k$. While the answer to this question is no, there exists a multivariate copula function, $C_{k}\left(F_{1}\left(x_{1}\right), F_{2}\left(x_{2}\right), \ldots, F_{k}\left(x_{k}\right)\right)$ from the family of extreme value copulas, which captures arbitrary values for the positive upper-tail dependencies among the random variables $X_{1}, X_{2}, \ldots, X_{k}$, while modeling the positive lower-tail dependencies with limited flexibility:

$$
\begin{aligned}
& \Theta\left(-\sum_{i=1}^{k} \sum_{j>i}^{k} \log K_{i, j}\left(e^{-\Theta^{-1}\left(F_{i}\left(x_{i}\right)\right) /\left(\vartheta_{i}+k-1\right)},\right.\right. \\
& \left.\left.e^{-\Theta^{-1}\left(F_{j}\left(x_{j}\right)\right) /\left(\vartheta_{j}+k-1\right)}\right)+\sum_{i=1}^{k} \frac{\vartheta_{i}}{\vartheta_{i}+k-1} \Theta^{-1}\left(F_{i}\left(x_{i}\right)\right)\right) .
\end{aligned}
$$

For this representation, Joe [26] provides the interpretation that Laplace transformation $\Theta$ represents a minimal level of pairwise global dependence, bivariate copula $K_{i, j}$ adds individual pairwise dependence beyond the global dependence, and parameters $\vartheta_{i}, i=1,2, \ldots, k$ lead to bivariate and multivariate asymmetry. Usually, the $\vartheta_{i}, i=1,2, \ldots, k$ are nonnegative, although they can be negative if some of the copulas $K_{i, j}$ correspond to independence. What is important to recognize here is that the Laplace transformation $\Theta$ limits our ability to represent arbitrary values for $v_{L}(i, j)>0$ and $v_{U}(i, j)>0$. This can be shown by selecting the Galambos copula for $K_{i, j}$; i.e.,

$$
\begin{aligned}
& K_{i, j}\left(u_{i}, u_{j} ; \theta_{i, j}\right) \\
& \quad=u_{i} u_{j} \exp \left(\left(\left(-\log u_{i}\right)^{-\theta_{i, j}}+\left(-\log u_{j}\right)^{-\theta_{i, j}}\right)^{-1 / \theta_{i, j}}\right)
\end{aligned}
$$

with $0 \leq \theta_{i, j}<\infty$ [84], and by choosing the Laplace transformation $\Theta$ as gamma type; i.e., $\Theta(s)=(1+s)^{-1 / \delta}$ with parameter $\delta \geq 0$. In this case, we obtain an upper-tail dependence of

$v_{U}(i, j)=\left(\left(\vartheta_{i}+k-1\right)^{\theta_{i, j}}+\left(\vartheta_{j}+k-1\right)^{\theta_{i, j}}\right)^{-1 / \theta_{i, j}}$

and a lower-tail dependence of

$v_{L}(i, j)=\left(2-\left(\left(\vartheta_{i}+k-1\right)^{\theta_{i, j}}+\left(\vartheta_{j}+k-1\right)^{\theta_{i, j}}\right)^{-1 / \theta_{i, j}}\right)^{-1 / \delta}$

for $i=1,2, \ldots, k$ and $j=i, i+1, \ldots, k$. The parameter $\theta_{i, j}$ is specific to the joint distribution of random variables $X_{i}$ and $X_{j}$, while global parameter $\delta$ is shared among all $k$ components $X_{i}, i=1,2, \ldots, k$. Therefore, we can represent any arbitrary value of upper-tail dependence between any pair of component random variables, but the global parameter $\delta$ limits our ability to have the same level of flexibility in representing lower-tail dependence.

To conclude, the use of mixtures of max-infinitely divisible copulas as well as multivariate Archimedean copulas for input modeling allows the representation of both the lower-tail dependence and the upper-tail dependence among the components of the random vector. However, the dependence structures captured by these copula families are restricted by the use of insufficient number of copula parameters. Although this particular limitation is overcome by the vine copula we present in the following section, the copula-vine method characterizes the dependence structure of the random vector using a mix of bivariate tail dependencies and bivariate conditional tail dependencies. Thus, in low-dimensional settings the simulation practitioner, who is interested in generating random vectors with pre-specified (unconditional) pair-wise 
lower-tail and upper-tail dependencies, might find the use of the mixtures of max-infinitely divisible copulas of this section more convenient than the vines of the following section.

\subsubsection{Vines}

A vine is a graphical model introduced in [85], studied extensively in [86-89], and described comprehensively in [90] for constructing multivariate distributions using a mix of bivariate and conditional bivariate distributions of uniform random variables. Specifically, a $k$-dimensional vine is a nested set of $k-1$ spanning trees where the edges of tree $j$ are the nodes of tree $j+1$, starting with a tree on a graph whose nodes are the $k$ component random variables $X_{i}, i=1,2, \ldots, k$. A regular vine is, on the other hand, a vine in which two edges in tree $j$ are joined by an edge in tree $j+1$ only if they share a common node [90, Section 4.4].

Fig. 3 presents a four-dimensional vine, which is a nested set of three spanning trees. Specifically, the first tree of this regular vine is the collection of three bivariate distributions; i.e., the joint distribution of the component random variables $X_{1}$ and $X_{2}$, the joint distribution of $X_{2}$ and $X_{3}$, and the joint distribution of $X_{3}$ and $X_{4}$. Since the solid line between $X_{1}$ and $X_{2}$ links these random variables, we associate this solid line with the joint distribution of $X_{1}$ and $X_{2}$, and label it as "1, 2", implying the copula density function $c_{2}\left(F_{1}\left(x_{1}\right), F_{2}\left(x_{2}\right)\right)$. Similarly, we denote the solid line between $X_{2}$ and $X_{3}$ by " 2,3 " associated with the copula density function $c_{2}\left(F_{2}\left(x_{2}\right), F_{3}\left(x_{3}\right)\right)$, and use " 3,4 " for the line between $X_{3}$ and $X_{4}$ to correspond to the copula density function $c_{2}\left(F_{3}\left(x_{3}\right), F_{4}\left(x_{4}\right)\right)$. The second tree, on the other hand, contains two bivariate distributions, which are the joint distribution of $X_{1} \mid X_{2}$ and $X_{3} \mid X_{2}$ and the joint distribution of $X_{2} \mid X_{3}$ and $X_{4} \mid X_{3}$ with the respective copula density functions $c_{2}\left(F_{1 \mid 2}\left(x_{1} \mid x_{2}\right), F_{3 \mid 2}\left(x_{3} \mid x_{2}\right)\right)$ and $c_{2}\left(F_{2 \mid 3}\left(x_{2} \mid x_{3}\right), F_{4 \mid 3}\left(x_{4} \mid x_{3}\right)\right)$. Therefore, in Fig. 3 we mark the lines "..." of the second tree by " $1,3 \mid 2$ " and " $2,4 \mid 3$ ". Finally, the third tree corresponds to the joint distribution of $X_{1} \mid X_{2}, X_{3}$ and $X_{4} \mid X_{2}, X_{3}$ represented by the line "-..-", which is further labeled by " $1,4 \mid 2,3$ " implying the copula density function $c_{2}\left(F_{1 \mid 2,3}\left(x_{1} \mid x_{2}, x_{3}\right), F_{4 \mid 2,3}\left(x_{4} \mid x_{2}, x_{3}\right)\right)$.

The two edges of a tree in Fig. 3 are joined only if they share a common component random variable to obtain an edge of the following tree. For example, the edges " 1,2 " and " 2 , 3" of the first tree share the node associated with the random variable $X_{2}$ and they are combined for the edge " $1,3 \mid 2$ " of the second tree, while the edge " $2,4 \mid 3$ " of the second tree is obtained from the edges " 2,3 " and " 3,4 " sharing the node of the random variable $X_{3}$. Similarly, the edges " $1,3 \mid 2$ " and " $2,4 \mid 3$ ", which share the nodes associated with the random variables $X_{2}$ and $X_{3}$ in the second tree, are joined by the edge " $1,4 \mid 2,3$ " of the third tree in a manner that is consistent with the definition of a regular vine.

More specifically, the regular vine in Fig. 3 is known as the drawable vine ( $\mathcal{D}$-vine). Its use for multivariate input modeling allows the four-dimensional copula density function to be represented by the product of the six bivariate linking copulas illustrated in Fig. 3; i.e.,

$$
\begin{aligned}
c_{4}( & \left.F_{1}\left(x_{1}\right), F_{2}\left(x_{2}\right), F_{3}\left(x_{3}\right), F_{4}\left(x_{4}\right)\right) \\
= & c_{2}\left(F_{1}\left(x_{1}\right), F_{2}\left(x_{2}\right)\right) \times c_{2}\left(F_{2}\left(x_{2}\right), F_{3}\left(x_{3}\right)\right) \\
& \times c_{2}\left(F_{3}\left(x_{3}\right), F_{4}\left(x_{4}\right)\right) \times c_{2}\left(F_{1 \mid 2}\left(x_{1} \mid x_{2}\right), F_{3 \mid 2}\left(x_{3} \mid x_{2}\right)\right) \\
& \times c_{2}\left(F_{2 \mid 3}\left(x_{2} \mid x_{3}\right), F_{4 \mid 3}\left(x_{4} \mid x_{3}\right)\right) \\
& \times c_{2}\left(F_{1 \mid 2,3}\left(x_{1} \mid x_{2}, x_{3}\right), F_{4 \mid 2,3}\left(x_{4} \mid x_{2}, x_{3}\right)\right) .
\end{aligned}
$$

The bivariate linking copulas of the first row appear in the first tree of Fig. 3, while the bivariate linking copulas of the next two rows come from the second and third trees. Furthermore, this characterization of the four-dimensional random vector is easily generalized to a $k$-dimensional random vector; i.e., the joint

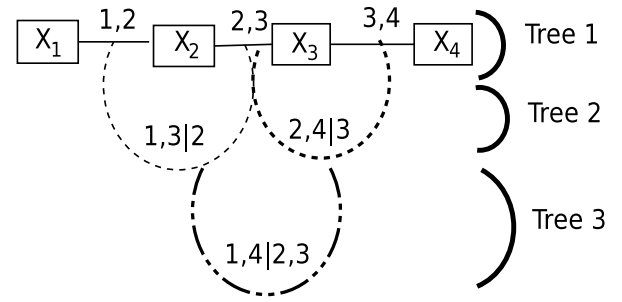

Fig. 3. A $\mathcal{D}$-vine specification on four dependent random variables.

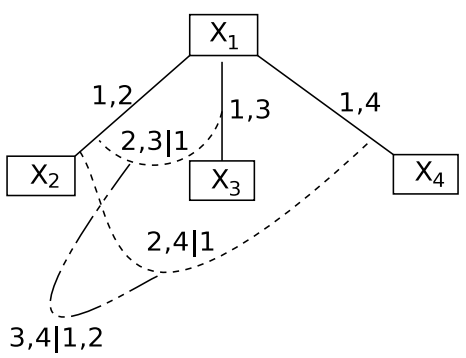

Fig. 4. A $\mathcal{C}$-vine specification on four dependent random variables.

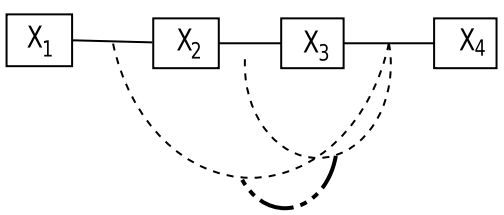

Fig. 5. An example of a non-regular vine on four dependent random variables.

density function of the $k$-dimensional $\mathcal{D}$-vine copula is obtained as a factorization of the univariate marginal density functions $\prod_{i=1}^{k} f_{i}\left(x_{i}\right)$ and the product of the bivariate (unconditional) linking copulas

$\prod_{i=1}^{k-1} c_{2}\left(F_{i}\left(x_{i}\right), F_{i+1}\left(x_{i+1}\right)\right)$

of the first tree and the bivariate (conditional) linking copulas

$$
\begin{gathered}
\prod_{j=2}^{k-1} \prod_{i=1}^{k-j} c_{2}\left(F_{i \mid i+1, \ldots, i+j-1}\left(x_{i} \mid x_{i+1}, \ldots, x_{i+j-1}\right),\right. \\
\left.F_{i+j \mid i+1, \ldots, i+j-1}\left(x_{i+j} \mid x_{i+1}, \ldots, x_{i+j-1}\right)\right)
\end{gathered}
$$

of the remaining $k-2$ trees. Representing the dependence structure of the $k$-dimensional random vector via these $(k-1)(k-$ 2 ) bivariate copulas instead of a single $k$-dimensional copula leads to computational tractability in the development of data-fitting algorithms in Section 4, goodness-of-fit tests in Section 5, and sampling procedures in Section 6 for copula-based multivariate input modeling.

Nevertheless, no unique regular vine exists for representing the dependence structure of a random vector. Fig. 4 presents another type of four-dimensional regular vine, the canonical vine ( $\mathcal{C}$-vine), which is often used for multivariate input modeling. Fig. 5, on the other hand, provides an example of a four-dimensional nonregular vine. The comparison of this vine to the regular vines in Figs. 3 and 4 shows that the dependence structure of the regular vine is represented in terms of unconditional and conditional dependence measures that are algebraically independent of each other; i.e., they do not need to satisfy any algebraic constraints for positive definiteness. Therefore, all assignments of the numbers between -1 and 1 to the edges of the regular vine are consistent 
in the sense that there is a joint distribution realizing these dependence measures.

A close look at the existing literature on vines reveals $\mathcal{C}$-vine and $\mathcal{D}$-vine to be the widely used regular vines for multi-dimensional dependence modeling. In recent years, the theory on vines has advanced considerably, accompanied by the development of several packages released by $\mathrm{R}$ software. In particular, we refer the reader to the recently released software packages CDVine, Vines, and Copula for statistical inference, distribution function evaluation, and simulation of $\mathcal{C}$-vine and $\mathcal{D}$-vine copulas. However, even if we narrow our choice of copulas to a $\mathcal{C}$-vine and a $\mathcal{D}$-vine, it is not clear which one of these vine structures would best capture the underlying dependence structure. Fortunately, this modeling challenge can be overcome by using the lattice-based algorithm of Maugis and Guegan [91] to find the vine copula representation that best models the dependence structure of the random vector of interest.

Joe et al. [92] show that vine copulas can cover a wide range of tail dependencies by choosing the bivariate linking copulas appropriately. However, the information set used to construct a vine copula is composed of a mix of unconditional bivariate tail dependencies and conditional bivariate tail dependencies. This raises the question of what the implied unconditional bivariate tail dependencies are for the given conditional tail dependencies. To explain this issue in detail, we consider the use of vines for constructing a three-dimensional random vector with components $X_{1}, X_{2}$, and $X_{3}$. If we are given bivariate joint cdfs $F\left(X_{1}, X_{2}\right)$ and $F\left(X_{2}, X_{3}\right)$ and the two-dimensional copula $C_{2}$ measuring the amount of conditional dependence between $X_{1} \mid X_{2}$ and $X_{3} \mid X_{2}$, then we first obtain the conditional marginal distributions $F_{1 \mid 2}$ and $F_{3 \mid 2}$ of the random variables $X_{1} \mid X_{2}$ and $X_{3} \mid X_{2}$, respectively, from $\partial F\left(x_{1}, x_{2}\right) / \partial x_{2}$ and $\partial F\left(x_{2}, x_{3}\right) / \partial x_{2}$. Then, we use these conditional marginal distributions and the copula $C_{2}$ together with the marginal distribution $F_{2}$ of the random variable $X_{2}$ to build the three-dimensional distribution

$F\left(x_{1}, x_{2}, x_{3}\right)=\int_{-\infty}^{x_{2}} C_{2}\left(F_{1 \mid 2}\left(x_{1} \mid x\right), F_{3 \mid 2}\left(x_{3} \mid x\right)\right) d F_{2}(x)$.

While the tail dependence parameters are explicitly chosen for the bivariate copulas, which link the random variables $X_{1}$ and $X_{2}$, the random variables $X_{2}$ and $X_{3}$, and the conditional random variables $X_{1} \mid X_{2}$ and $X_{3} \mid X_{2}$, the tail dependence between random variables $X_{1}$ and $X_{3}$ are not explicitly defined, even though it can be obtained from the joint cdf $F$. Under certain regularity conditions, Joe [26] proves the existence of an upper-tail dependence between $X_{1}$ and $X_{3}$ when both the bivariate copula linking $X_{1}$ and $X_{2}$ and the bivariate copula linking $X_{2}$ and $X_{3}$ have upper-tail dependencies. This result is further shown to hold for a $k$-dimensional random vector [92].

An alternative to the representation of the dependence structure of a multivariate random vector in terms of bivariate tail dependencies is to measure the multivariate tail dependence. Joe et al. [92] define the lower-tail dependence of the $k$ dimensional copula $C_{k}$ as $\lim _{u \downarrow 0} C_{k}(u, u, \ldots, u) / u$ and the uppertail dependence as $\lim _{u \downarrow 0} \bar{C}_{k}(1-u, 1-u, \ldots, 1-u) / u$ with $\bar{C}_{k}$ the survival function of $C_{k}$. It is shown that the multivariate tail dependence function of a vine copula can be expressed recursively by the tail dependence and conditional tail dependence functions of lower-dimensional margins. Also, a vine copula is identified as tail dependent if all the bivariate linking copulas associated with the first tree of the vine (i.e., baseline copulas) are tail dependent. More specifically, if the baseline copulas are all lower-tail (uppertail) dependent, then the vine copula is lower-tail (upper-tail) dependent provided that $[0,1]^{2}$ is the support of the bivariate linking copulas. However, if some of the baseline copulas are tail independent, then the vine copula is tail independent.

\section{Fitting methods}

In this section, we assume the availability of multivariate input data and review the methods of parameter estimation for the copulas of Section 3. Specifically, Section 4.1 discusses the use of frequentist methods (i.e., the methods that only rely on the available input data), while Section 4.2 reviews the limited literature on Bayesian methods (i.e., the methods that combine the input data with the prior information available about the joint distribution parameters) for copula-parameter estimation.

\subsection{Frequentist method}

This section reviews the methods of Maximum Likelihood Estimation (MLE) and the Inference For Margins (IFM) to estimate the parameters of the copula-based input models.

The application of the MLE method for parameter estimation requires the maximization of the joint likelihood function of the available input data $x_{i, t}, i=1,2, \ldots, k, t=1,2, \ldots, n$ of length $n$ with respect to the parameters of the joint density function. The utilization of Sklar's marginal-copula representation for the joint density function of the $k$-dimensional random vector $\mathbf{X}=\left(X_{1}, X_{2}\right.$, $\left.\ldots, X_{k}\right)^{\prime}$ with marginal-distribution parameter vectors $\boldsymbol{\Psi}_{i}, i=1$, $2, \ldots, k$ and the copula parameter vector $\Upsilon$ leads to the following log-likelihood function, where $\mathbf{x}$ denotes the vector of the available input data:

$$
\begin{aligned}
\mathcal{L}\left(\boldsymbol{\Psi}_{1}, \boldsymbol{\Psi}_{2}, \ldots, \boldsymbol{\Psi}_{k}, \mathbf{\Upsilon} \mid \mathbf{x}\right)= & \sum_{i=1}^{k} \sum_{t=1}^{n} \log f_{i}\left(x_{i, t} ; \boldsymbol{\Psi}_{i}\right) \\
& +\sum_{t=1}^{n} \log c_{k}\left(F_{1}\left(x_{1, t} ; \boldsymbol{\Psi}_{1}\right),\right. \\
& \left.F_{2}\left(x_{2, t} ; \boldsymbol{\Psi}_{2}\right), \ldots, F_{k}\left(x_{k, t} ; \boldsymbol{\Psi}_{k}\right) ; \mathbf{\Upsilon}\right) .
\end{aligned}
$$

The maximum likelihood estimators $\tilde{\boldsymbol{\Psi}}_{i}^{\mathrm{MLE}}, i=1,2, \ldots, k$, and $\tilde{\Upsilon}^{\mathrm{MLE}}$ are obtained from the maximization of this log-likelihood function; i.e.,

$$
\begin{aligned}
& \left(\tilde{\boldsymbol{\Psi}}_{1}^{\mathrm{MLE}}, \tilde{\boldsymbol{\Psi}}_{2}^{\mathrm{MLE}}, \ldots, \tilde{\boldsymbol{\Psi}}_{k}^{\mathrm{MLE}}, \tilde{\boldsymbol{\Upsilon}}^{\mathrm{MLE}}\right)^{\prime} \\
& =\underset{\boldsymbol{\Psi}_{1}, \boldsymbol{\Psi}_{2}, \ldots, \boldsymbol{\Psi}_{k}, \Upsilon}{\arg \max } \mathcal{L}\left(\boldsymbol{\Psi}_{1}, \boldsymbol{\Psi}_{2}, \ldots, \boldsymbol{\Psi}_{k}, \mathbf{\Upsilon} \mid \mathbf{x}\right) .
\end{aligned}
$$

Under the regularity conditions of the asymptotic likelihood theory [93], $\tilde{\mathbf{\Psi}}_{i}^{\mathrm{MLE}}, i=1,2, \ldots, k$, and $\tilde{\boldsymbol{\Upsilon}}^{\mathrm{MLE}}$ are strongly consistent and asymptotically normal; i.e., as $n \rightarrow \infty$, the random vector $\sqrt{n}\left(\left(\tilde{\boldsymbol{\Psi}}_{1}^{\mathrm{MLE}}, \tilde{\boldsymbol{\Psi}}_{2}^{\mathrm{MLE}}, \ldots, \tilde{\boldsymbol{\Psi}}_{k}^{\mathrm{MLE}}, \tilde{\boldsymbol{\Upsilon}}^{\mathrm{MLE}}\right)^{\prime}-\left(\boldsymbol{\Psi}_{1}^{*}, \boldsymbol{\Psi}_{2}^{*}, \ldots, \boldsymbol{\Psi}_{k}^{*}, \mathbf{\Upsilon}^{*}\right)^{\prime}\right)$ converges to be normally distributed with the zero mean vector and the variance-covariance matrix $\tau^{-1}\left(\boldsymbol{\Psi}_{1}^{*}, \boldsymbol{\Psi}_{2}^{*}, \ldots, \boldsymbol{\Psi}_{k}^{*}, \boldsymbol{\Upsilon}^{*}\right)$, where $\boldsymbol{\Psi}_{i}^{*}, i=1,2, \ldots, k$, and $\boldsymbol{\Upsilon}^{*}$ are the true marginal-distribution and copula parameter vectors and $\tau\left(\boldsymbol{\Psi}_{1}^{*}, \boldsymbol{\Psi}_{2}^{*}, \ldots, \boldsymbol{\Psi}_{k}^{*}, \mathbf{\Upsilon}^{*}\right)$ is the Fisher's information matrix [94].

Despite the wide use of the MLE method for parameter estimation, the simultaneous estimation of the marginal-distribution parameters and the copula parameters gets computationally demanding with $k$, the number of component random variables. Fortunately, the log-likelihood function $\mathcal{L}\left(\boldsymbol{\Psi}_{1}, \boldsymbol{\Psi}_{2}, \ldots, \boldsymbol{\Psi}_{k}, \mathbf{\Upsilon} \mid \mathbf{x}\right)$ can be partitioned into terms that separately relate to the component marginal density functions and the copula density function. This makes it possible to estimate the marginal distribution parameters and the copula parameters in two stages. The resulting method of parameter estimation is known as the IFM method [26, Chapter 10]. First, we independently estimate the marginal distribution parameters $\tilde{\Psi}_{i}^{\mathrm{IFM}}, i=1,2, \ldots, k$ from the log-likelihood functions associated with the marginal distributions 
$\mathcal{L}_{i}\left(\boldsymbol{\Psi}_{i} \mid \mathbf{x}_{i}\right)=\sum_{t=1}^{n} \log f_{i}\left(x_{i, t} ; \boldsymbol{\Psi}_{i}\right), i=1,2, \ldots, k$; i.e., $\tilde{\mathbf{\Psi}}_{i}^{\mathrm{IFM}}=$ $\arg \max _{\Psi_{i}} \mathcal{L}_{i}\left(\boldsymbol{\Psi}_{i} \mid \mathbf{x}_{i}\right)$ for $i=1,2, \ldots, k$. Then, we estimate the copula parameter vector $\tilde{\Upsilon}^{\mathrm{IFM}}$ from the part of the log-likelihood function that is associated with the copula density function; i.e.,

$$
\begin{aligned}
& \mathcal{L}_{c}\left(\boldsymbol{\Upsilon} \mid \boldsymbol{\Psi}_{1}, \boldsymbol{\Psi}_{2}, \ldots, \boldsymbol{\Psi}_{k}, \mathbf{x}\right) \\
& \quad=\sum_{t=1}^{n} \log c_{k}\left(F_{1}\left(x_{1, t} ; \boldsymbol{\Psi}_{1}\right), F_{2}\left(x_{2, t} ; \boldsymbol{\Psi}_{2}\right), \ldots, F_{k}\left(x_{k, t} ; \boldsymbol{\Psi}_{k}\right) ; \boldsymbol{\Upsilon}\right),
\end{aligned}
$$

by using the marginal distribution parameter estimates $\tilde{\boldsymbol{\Psi}}_{i}^{\mathrm{IFM}}$, $i=1,2, \ldots, k$ obtained in the first stage; i.e., $\tilde{\Upsilon}^{\mathrm{IFM}}=\arg \max _{\Upsilon} \mathcal{L}_{c}$ $\left(\mathbf{\Upsilon} \mid \tilde{\boldsymbol{\Psi}}_{1}^{\mathrm{IFM}}, \tilde{\boldsymbol{\Psi}}_{2}^{\text {IFM }}, \ldots, \tilde{\boldsymbol{\Psi}}_{k}^{\text {IFM }}, \mathbf{x}\right)$. However, the use of a copula-vine specification to construct a $k$-dimensional input model as a nested set of $k-1$ spanning trees further decomposes the estimation of the copula parameter vector into $k-1$ stages.

The IFM estimators $\tilde{\Psi}_{i}^{\mathrm{IFM}}, i=1,2, \ldots, k$, and $\tilde{\Upsilon}^{\mathrm{IFM}}$ are generally not equivalent to the MLE estimators $\tilde{\boldsymbol{\Psi}}_{i}^{\mathrm{MLE}}, i=1,2, \ldots, k$, and $\tilde{\Upsilon}^{\mathrm{MLE}}$ except for the normal copula with the normally distributed marginal distributions. Nevertheless, Joe [26] proves that the IFM estimators are also strongly consistent and asymptotically normal under certain regularity conditions; i.e., $\sqrt{n}\left(\left(\tilde{\boldsymbol{\Psi}}_{1}^{\mathrm{IFM}}, \tilde{\mathbf{\Psi}}_{2}^{\mathrm{IFM}}\right.\right.$, $\left.\left.\ldots, \tilde{\boldsymbol{\Psi}}_{k}^{\mathrm{IFM}}, \tilde{\boldsymbol{\Upsilon}}^{\mathrm{IFM}}\right)^{\prime}-\left(\boldsymbol{\Psi}_{1}^{*}, \boldsymbol{\Psi}_{2}^{*}, \ldots, \boldsymbol{\Psi}_{k}^{*}, \boldsymbol{\Upsilon}^{*}\right)^{\prime}\right) \rightarrow \mathbf{N}\left(\mathbf{0}, \mathbf{G}^{-1}\left(\boldsymbol{\Psi}_{1}^{*}, \boldsymbol{\Psi}_{2}^{*}\right.\right.$, $\left.\left.\ldots, \boldsymbol{\Psi}_{k}^{*}, \boldsymbol{\Upsilon}^{*}\right)\right)$ with $\mathbf{G}\left(\boldsymbol{\Psi}_{1}, \boldsymbol{\Psi}_{2}, \ldots, \boldsymbol{\Psi}_{k}, \boldsymbol{\Upsilon}\right)$ the Godambe information matrix. If we define $\mathbf{S}\left(\boldsymbol{\Psi}_{1}, \boldsymbol{\Psi}_{2}, \ldots, \boldsymbol{\Psi}_{k}, \boldsymbol{\Upsilon}\right)$ as a vector whose transpose is the collection of $\nabla \boldsymbol{\Psi}_{i} \mathcal{L}_{i}\left(\boldsymbol{\Psi}_{i} \mid \mathbf{x}_{i}\right), i=1,2, \ldots, k$, and $\nabla \Upsilon \mathcal{L}_{c}\left(\boldsymbol{\Upsilon} \mid \boldsymbol{\Psi}_{1}, \boldsymbol{\Psi}_{2}, \ldots, \boldsymbol{\Psi}_{k}, \mathbf{x}\right)$, then the Godambe information matrix $\mathbf{G}\left(\boldsymbol{\Psi}_{1}, \boldsymbol{\Psi}_{2}, \ldots, \boldsymbol{\Psi}_{k}, \boldsymbol{\Upsilon}\right)$ is given by $\mathbf{D}^{-1} \mathbf{V}\left(\mathbf{D}^{-1}\right)^{\prime}$, where $\mathbf{D}=$ $\mathrm{E}\left(\nabla \mathbf{S}\left(\boldsymbol{\Psi}_{1}, \boldsymbol{\Psi}_{2}, \ldots, \boldsymbol{\Psi}_{k}, \boldsymbol{\Upsilon}\right) / \nabla\left(\boldsymbol{\Psi}_{1}, \boldsymbol{\Psi}_{2}, \ldots, \boldsymbol{\Psi}_{k}, \boldsymbol{\Upsilon}\right)\right)$ and $\mathbf{V}=\mathrm{E}(\mathbf{S}$ $\left.\left(\boldsymbol{\Psi}_{1}, \boldsymbol{\Psi}_{2}, \ldots, \boldsymbol{\Psi}_{k}, \boldsymbol{\Upsilon}\right) \mathbf{S}\left(\boldsymbol{\Psi}_{1}, \boldsymbol{\Psi}_{2}, \ldots, \boldsymbol{\Psi}_{k}, \boldsymbol{\Upsilon}\right)^{\prime}\right)$ [44]. However, it is well known that the estimators obtained from the entire loglikelihood function are the most efficient estimators, in that they attain the minimum asymptotic variance bound, while the estimators obtained from the multi-stage maximum-likelihood estimation do not attain this bound. Furthermore, an inappropriate choice of models for the marginal distributions may have detrimental effects on the estimation of the copula parameter vector [95]. Therefore, the IFM estimators $\tilde{\boldsymbol{\Psi}}_{i}^{\mathrm{IFM}}, i=1,2, \ldots, k$, and $\tilde{\Upsilon}^{\mathrm{IFM}}$ provide good starting points for obtaining more efficient estimators by solving $\mathcal{L}\left(\boldsymbol{\Psi}_{1}, \boldsymbol{\Psi}_{2}, \ldots, \boldsymbol{\Psi}_{k}, \mathbf{\Upsilon} \mid \mathbf{x}\right)$ for both the marginal-distribution parameter vectors $\boldsymbol{\Psi}_{i}, i=1,2, \ldots, k$ and the copula parameter vector $\Upsilon$ in a single stage.

\subsection{Bayesian method}

An alternative method to the use of the frequentist MLE and IFM methods for parameter estimation is the Bayesian method. The Bayesian method starts with the determination of a joint prior density function that quantifies the initial uncertainty about the multivariate distribution parameters. Then, the joint prior distribution is updated with the joint likelihood function of the available historical data, and we obtain the joint posterior distribution that captures the uncertainty associated with the multivariate distribution parameters. The derivation of the posterior density function generally requires the computation of high-dimensional integrals, limiting the use of the Bayesian method for parameter estimation in multi-dimensional settings. However, the advancement of the Markov Chain Monte Carlo (MCMC) method in recent years has made it possible to estimate distribution parameters with any posterior density function. In this section, we review Bayesian estimation using the MCMC method to obtain parameter estimates for three copula models: the multivariate normal copula, the multivariate $t$ copula, and the $\mathcal{D}$-vine built on bivariate $t$ copulas. Although the multivariate normal copula does not capture any tail dependence, we describe a Bayesian estimation for this copula due to its relation to the multivariate $t$ copula.

Dalla Valle [96] builds a Bayesian model to estimate the correlation matrix $\boldsymbol{\Sigma}_{k}$ of the $k$-dimensional normal copula density function with a likelihood function of the following form, where $\mathbf{x}_{t}$ is the vector of input data $x_{i, t}, i=1,2, \ldots, k$ and $\mathbf{I}_{k}$ is the $k$ dimensional identity matrix:

$\left|\boldsymbol{\Sigma}_{k}\right|^{-n / 2} \exp \left(-\frac{1}{2} \sum_{t=1}^{n} \mathbf{x}_{t}^{\prime}\left(\boldsymbol{\Sigma}_{k}^{-1}-\mathbf{I}_{k}\right) \mathbf{x}_{t}\right)$.

Dalla Valle [96] uses the Inverse Wishart density function as the conjugate prior for the correlation matrix $\boldsymbol{\Sigma}_{k}$; i.e., $\boldsymbol{\Sigma}_{k} \sim$ Inverse $\operatorname{Wishart}(\alpha, \mathbf{B})$ [97]. The parameters of this conjugate prior are chosen as $\alpha \equiv k+1$ and $\mathbf{B} \equiv\left[\operatorname{diag}\left(\gamma_{i}\right) ; i=1,2, \ldots, k\right]$, where $\gamma_{i}$ is the gamma random variable whose scale and shape parameters are 0.001 to minimize the impact of the prior density function on the posterior density function. Therefore, the posterior density function is identified as the Inverse Wishart with parameters $n / 2+\alpha$ and $\mathbf{B}+\sum_{t=1}^{n} \mathbf{x}_{t} \mathbf{x}_{t}^{\prime} / 2$. Dalla Valle [96] obtains an estimate of $\boldsymbol{\Sigma}_{k}$ from this posterior density function via the use of a Gibbs sampler algorithm, which is a widely used MCMC method that requires the sampling of $\Sigma_{k}$ from its conditional posterior density function. The key idea behind any MCMC method is to simulate a random walk on the entire parameter space that converges to the joint posterior density function of the parameters [98]. Then, the parameters sampled in each replication of the Gibbs sampler algorithm are averaged to estimate the copula parameters.

Another distribution Dalla Valle [96] considers is the $k$ dimensional $t$ copula with a likelihood function given by

$$
\begin{aligned}
& {\left[\Gamma\left(\frac{d+k}{2}\right)\right]^{n}\left[\Gamma\left(\frac{d+1}{2}\right)\right]^{-k n}\left[\Gamma\left(\frac{d}{2}\right)\right]^{n(k-1)}} \\
& \quad \times\left|\Sigma_{k}\right|^{-n / 2} \prod_{t=1}^{n}\left[1+\frac{\mathbf{x}_{t}^{\prime} \mathbf{\Sigma}_{k}^{-1} \mathbf{x}_{t}}{d}\right]^{-\frac{d+k}{2}} \prod_{t=1}^{n} \prod_{i=1}^{k}\left[1+\frac{x_{i t}^{2}}{d}\right]^{\frac{d+1}{2}} .
\end{aligned}
$$

Similar to the selection of a joint prior density function for the normal copula parameters, Dalla Valle chooses the Inverse Wishart prior with parameters $\alpha$ and $\mathbf{B}$ for $\boldsymbol{\Sigma}_{k}$ of the $k$-dimensional $t$ copula. Additionally, the truncated Poisson distribution with parameter $h$ is selected as a prior for $d$. Consequently, the posterior density function of $\boldsymbol{\Sigma}_{k}$ is obtained as

$$
\left|\boldsymbol{\Sigma}_{k}\right|^{-\frac{n}{2}-\alpha+\frac{k+1}{2}} \prod_{t=1}^{n}\left[1+\frac{\mathbf{x}_{t}^{\prime} \boldsymbol{\Sigma}_{k}^{-1} \mathbf{x}_{t}}{d}\right]^{-\frac{d+k}{2}} \exp \left[-\operatorname{tr}\left(\mathbf{B} \boldsymbol{\Sigma}_{k}^{-1}\right)\right],
$$

while the posterior density function of $d$ is given by

$$
\begin{aligned}
& {\left[\Gamma\left(\frac{d+k}{2}\right)\right]^{n}\left[\Gamma\left(\frac{d+1}{2}\right)\right]^{-k n}\left[\Gamma\left(\frac{d}{2}\right)\right]^{n(k-1)}} \\
& \times \prod_{t=1}^{n}\left[1+\frac{\mathbf{x}_{t}^{\prime} \boldsymbol{\Sigma}_{k}^{-1} \mathbf{x}_{t}}{d}\right]^{\frac{d+k}{2}} \prod_{t=1}^{n} \prod_{i=1}^{k}\left[1+\frac{x_{i t}^{2}}{d}\right]^{\frac{d+1}{2}} \frac{h^{d}}{d !} .
\end{aligned}
$$

Both of these posterior density functions are in non-standard forms; therefore, Dalla Valle [96] resorts to the use of an MCMC method known as the Metropolis Hastings algorithm for estimating $\boldsymbol{\Sigma}_{k}$ and $d$. The implementation details of this algorithm can be found in [98].

Czado and Min [99], on the other hand, consider the Bayesian estimation of the parameters of a $\mathcal{D}$-vine built on bivariate $t$ copulas with the following key assumption: The degrees of freedom parameter, the dependence parameters of the baseline 
copulas, and the conditional dependence parameters of the conditional bivariate linking copulas are independent. Czado and Min use a uniform prior in the interval $(1,100)$ for the degrees of freedom parameter and a uniform prior in the interval $(-1,1)$ for each of the dependence parameters. However, the resulting joint posterior density function of the copula parameters does not have a well-defined form; therefore, the authors also use the Metropolis Hastings algorithm for estimating the vine copula parameters. Details of implementing this algorithm for the $\mathcal{D}$-vine are available in [99].

\section{Goodness-of-fit tests}

The next step in multivariate input modeling is to assess the goodness of the estimated copula parameters in capturing the joint distributional characteristics of the available input data. A close look at the existing literature reveals several goodness-of-fit tests that have been built specifically for copulas; we refer the reader to Berg [100] and Genest et al. [101] for a comprehensive review as well as a comparison of these tests. In this section, we review only two of these tests; i.e., a test that is based on the empirical copula and a test proposed by Genest et al. [101] building on a variation of Rosenblatt's transformation. The reason for considering only these tests in our survey is that they work for any copula function and they are not sensitive to the grouping of the data. Genest et al. [101] call the tests with such characteristics the "blanket" tests. Among all blanket tests considered in [101], the ones that are reviewed here standout as the best blanket tests that have been developed for copula models.

To focus our presentation on the fit of the copula itself and avoid any distributional assumptions about the component marginal distributions, we consider the ranks of the historical data in this section and denote them by $b_{i, t}, i=1,2, \ldots, k, t=1,2, \ldots$, $n$. Therefore, we define a pseudo-observation, $u_{i, t}$ by $b_{i, t} /(n+1)$. The pseudo-observations $u_{i, t}, i=1,2, \ldots, k, t=1,2, \ldots, n$ can be interpreted as a sample from the underlying $k$-dimensional copula $C_{k}$, whose empirical counterpart $C_{k, n}(\mathbf{u})$ with $\mathbf{u}=\left(u_{1}, u_{2}\right.$, $\left.\ldots, u_{k}\right)^{\prime} \in[0,1]^{k}$ is given by

$C_{k, n}(\mathbf{u})=\frac{1}{n} \sum_{t=1}^{n} \mathcal{I}\left(u_{1, t} \leq u_{1}, u_{2, t} \leq u_{2}, \ldots, u_{k, t} \leq u_{k}\right)$,

where $\mathcal{I}$ represents the indicator function that equals 1 if its argument is true, and 0 otherwise [102]. It is important to note that the pseudo-observations are not mutually independent and they are only approximately uniform on $[0,1]$. Any inference procedure based on the pseudo-observations should take these features into account. A detailed discussion about the significance of these properties of the pseudo-observations on the performance of the goodness-of-fit tests is available in [101].

Building on the empirical copula $C_{k, n}$, Genest and Rémillard [103] propose the use of the rank-based version of Cramér von Mises test statistic,

$S_{n}=\int_{[0,1]^{k}} n\left(C_{k, n}(\mathbf{u})-C_{k}\left(\mathbf{u} ; \hat{\Upsilon}_{n}\right)\right)^{2} d C_{k, n}(\mathbf{u})$

and the rank-based version of the Kolmogorov-Smirnov test statistic,

$T_{n}=\sup _{\mathbf{u} \in[0,1]^{k}}\left|\sqrt{n}\left(C_{k, n}(\mathbf{u})-C_{k}\left(\mathbf{u} ; \hat{\Upsilon}_{n}\right)\right)\right|$,

where $C_{k}\left(\cdot ; \hat{\Upsilon}_{n}\right)$ denotes the $k$-dimensional copula with the dependence parameter vector $\hat{\Upsilon}_{n}$ estimated from multivariate data of length $n$. Small values of $S_{n}$ and $T_{n}$ indicate the goodness of the copula fit. The approximate $p$ values of these tests can be obtained from their limiting distributions. However, in practice the limiting distributions of $S_{n}$ and $T_{n}$ depend on the copula family of interest and the unknown dependence parameter vector $\Upsilon$. Therefore, the common practice is to obtain the asymptotic distribution of these tests and the approximate $p$ values via Monte Carlo methods [101]. A parametric bootstrap procedure, which can be used for this purpose, is available in Appendix A of Genest et al. [101], while the consistency and the asymptotic convergence of these tests are proven in [103].

The second goodness-of-fit test we present for copula-based input modeling is based on Rosenblatt's transformation [104], which is a general procedure used in simulation for decomposing a random vector with a given distribution into mutually independent components that are uniformly distributed on the unit interval. Specifically, Rosenblatt [104] transforms the random vector $\mathbf{U}=\left(U_{1}, U_{2}, \ldots, U_{k}\right)^{\prime} \in[0,1]^{k}$ to the random vector $\mathcal{R}(\mathbf{U})=$ $\left(Y_{1}, Y_{2}, \ldots, Y_{k}\right)^{\prime} \in[0,1]^{k}$ with $Y_{1}=U_{1}$ and

$Y_{i}=\frac{\left(\partial^{i-1} C_{k}\left(U_{1}, U_{2}, \ldots, U_{i-1}, U_{i}, 1, \ldots, 1 ; \Upsilon\right)\right) /\left(\partial U_{1} \partial U_{2} \cdots \partial U_{i-1}\right)}{\left(\partial^{i-1} C_{k}\left(U_{1}, U_{2}, \ldots, U_{i-1}, 1,1, \ldots, 1 ; \Upsilon\right)\right) /\left(\partial U_{1} \partial U_{2} \cdots \partial U_{i-1}\right)}$

for $i=2,3, \ldots, k$. The use of this probability integral transform of the $k$-dimensional copula $C_{k}(\cdot ; \Upsilon)$ for measuring the goodness of a copula fit leads to the interpretation that the pseudo-observations $\mathbf{y}_{t}=\left(y_{1, t}, y_{2, t}, \ldots, y_{k, t}\right)^{\prime}, t=1,2, \ldots, n$, which are obtained from $\mathcal{R}\left(\mathbf{u}_{t}\right), t=1,2, \ldots, n$ with $\mathbf{u}_{t}=\left(u_{1, t}, u_{2, t}, \ldots, u_{k, t}\right)^{\prime}$, correspond to a sample from the independence copula $C_{\perp}(\mathbf{u})$ (i.e., $\left.C_{\perp}\left(u_{1}, u_{2}, \ldots, u_{k}\right)=u_{1} u_{2} \cdots u_{k}\right)[101]$.

Building on this result, Genest et al. [101] devise two tests that are based on the empirical distribution function

$D_{k, n}(\mathbf{u})=\frac{1}{n} \sum_{t=1}^{n} \mathcal{I}\left(\mathbf{y}_{t} \leq \mathbf{u}\right)$

associated with the pseudo-observations $\mathbf{y}_{t}, t=1,2, \ldots, n$. Any discrepancy between this empirical copula $\left(D_{k, n}(\mathbf{u})\right)$ and the independence copula $\left(C_{\perp}(\mathbf{u})\right)$ is interpreted as an indication of a lack of fit. The statistics of the resulting Cramér von Mises tests are as follows:

$$
\begin{aligned}
S_{n}^{(B)}= & n \int_{[0,1]^{k}}\left(D_{k, n}(\mathbf{u})-C_{\perp}(\mathbf{u})\right)^{2} d \mathbf{u}, \\
= & \frac{n}{3^{k}}-\frac{1}{2^{k-1}} \sum_{t=1}^{n} \prod_{i=1}^{k}\left(1-y_{i, t}^{2}\right) \\
& +\frac{1}{n} \sum_{s=1}^{n} \sum_{t=1}^{n} \prod_{i=1}^{k}\left(1-\max \left(y_{i, s}, y_{i, t}\right)\right) . \\
S_{n}^{(C)}= & n \int_{[0,1]^{k}}\left(D_{k, n}(\mathbf{u})-C_{\perp}(\mathbf{u})\right)^{2} d D_{k, n}(\mathbf{u}), \\
= & \sum_{t=1}^{n}\left(D_{k, n}\left(\mathbf{y}_{t}\right)-C_{\perp}\left(\mathbf{y}_{t}\right)\right)^{2} .
\end{aligned}
$$

We refer the reader to Appendix D of Genest et al. [101] for a parametric bootstrap procedure using the test statistics $S_{n}^{(B)}$ and $S_{n}^{(C)}$ to measure the goodness of a copula fit.

A comprehensive experimental study conducted by Genest et al. [101] shows that the test statistics based on the Cramér-von Mises distance perform better than the test statistics based on the Kolmogorov-Smirnov distance; i.e., the test statistics $S_{n}, S_{n}^{(B)}$, and $S_{n}^{(C)}$ outperform the test statistic $T_{n}$. Furthermore, among the test statistics based on Cramér-von Mises distance, $S_{n}$ and $S_{n}^{(B)}$ are the most promising tests for various copula models including the normal copula, the $t$ copula, the Clayton copula, and the Gumbel copula. Recent applications of these test statistics to financial data can be found in [105]. 


\section{Sampling}

We discuss the well known methods of generating random vectors from multivariate normal and $t$ copulas in Section 6.1, from Archimedean copulas via conditional sampling in Section 6.2, and from $\mathcal{C}$-vine and $\mathcal{D}$-vine specifications in Section 6.3.

\subsection{Sampling from the normal copula and the t copula}

The well known approach to the sampling of a uniform random vector $\left(U_{1}, U_{2}, \ldots, U_{k}\right)^{\prime}$ from the $k$-dimensional normal copula with correlation matrix $\boldsymbol{\Sigma}_{k}$ is based on using the Cholesky decomposition for variate generation [106]. Specifically, we first find the Cholesky decomposition of the correlation matrix $\boldsymbol{\Sigma}_{k}$; i.e., $\boldsymbol{\Sigma}_{k}=\mathbf{A} \mathbf{A}^{\prime}$. Then, we generate $k$ independent standard normal random variates $z_{i}, i=1,2, \ldots, k$; construct vector $\mathbf{z}=$ $\left(z_{1}, z_{2}, \ldots, z_{k}\right)^{\prime}$; and set $\mathbf{x}=\left(x_{1}, x_{2}, \ldots, x_{k}\right)^{\prime}$ to $\mathbf{A z}$. Finally, we apply the transformation $u_{i}=\Phi\left(x_{i}\right)$ for $i=1,2, \ldots, k$.

We can similarly sample a uniform random vector from the $k$ dimensional $t$ copula with parameters $\boldsymbol{\Sigma}_{k}$ and $d$. First, we obtain the vector $\mathbf{x}$ as described above for the $k$-dimensional normal copula. Then, we generate a random variate $s$ from a chi-square distribution with $d$ degrees of freedom and set $\mathbf{m}=\sqrt{(d / s)} \mathbf{x}$. Finally, we obtain $u_{i}, i=1,2, \ldots, k$ from the transformation $t_{d}\left(m_{i}\right), i=1,2, \ldots, k$.

\subsection{Sampling from the Archimedean copulas}

The generation of a two-dimensional random vector of the form $\left(u_{1}, u_{2}\right)^{\prime}$ from the copula $C_{2}$ starts with the sampling of two independent uniform random variates $u_{1}$ and $w$. Then, we obtain the uniform random variate $u_{2}$ from the quasi-inverse function of the conditional distribution $\operatorname{Pr}\left(U_{2} \leq u_{2} \mid U_{1}=u_{1}\right)$; i.e., $u_{2}=C_{2 \mid 1}^{-1}\left(w \mid u_{1}\right)$. Specifically, we obtain the conditional copula $C_{2 \mid 1}\left(u_{2} \mid u_{1}\right)$ from $\partial C_{2}\left(u_{1}, u_{2}\right) / \partial u_{1}$. In the case of a $k$-dimensional setting with $k \geq 3$, we similarly generate $k$ independent uniform random variates, $u_{1}, w_{1}, w_{2}, \ldots, w_{k-1}$, and obtain the random variates $u_{2}, u_{3}, \ldots, u_{k}$ by

$u_{2}=C_{2 \mid 1}^{-1}\left(w_{1} \mid u_{1}\right)$,

$u_{3}=C_{3 \mid 1,2}^{-1}\left(w_{2} \mid u_{1}, u_{2}\right)$,

$u_{k}=C_{k \mid 1,2, \ldots, k-1}^{-1}\left(w_{k-1} \mid u_{1}, u_{2}, \ldots, u_{k-1}\right)$,

where $C_{k \mid 1,2, \ldots, k-1}\left(u_{k} \mid u_{1}, u_{2}, \ldots, u_{k-1}\right)=\operatorname{Pr}\left(U_{k} \leq u_{k} \mid U_{1}=\right.$ $\left.u_{1}, U_{2}=u_{2}, \ldots, U_{k-1}=u_{k-1}\right)$ is given by

$\frac{\left(\partial^{k-1} C_{k}\left(u_{1}, u_{2}, \ldots, u_{k}\right)\right) /\left(\partial u_{1} \ldots \partial u_{k-1}\right)}{\left(\partial^{k-1} C_{k-1}\left(u_{1}, u_{2}, \ldots, u_{k-1}\right)\right) /\left(\partial u_{1} \ldots \partial u_{k-1}\right)}$.

The functional form of the conditional copula for the $k$ dimensional Archimedean copula reduces to the following representation, where $\phi^{-1(i)}(\cdot ; \theta)$ stands for the $i$ th-order derivative of the pseudo-inverse of the generator function $\phi(\cdot ; \theta)[44]$ :

$$
\begin{aligned}
& C_{i \mid 1,2, \ldots, i-1}\left(u_{i} \mid u_{1}, u_{2}, \ldots, u_{i-1} ; \theta\right) \\
& \quad=\frac{\phi^{-1(i-1)}\left(\phi\left(u_{1} ; \theta\right)+\phi\left(u_{2} ; \theta\right)+\cdots+\phi\left(u_{i} ; \theta\right) ; \theta\right)}{\phi^{-1(i-1)}\left(\phi\left(u_{1} ; \theta\right)+\phi\left(u_{2} ; \theta\right)+\cdots+\phi\left(u_{i-1} ; \theta\right) ; \theta\right)}, \\
& \quad i=2,3, \ldots, k
\end{aligned}
$$

Therefore, we can sample from the $k$-dimensional Clayton copula with

$$
\phi^{-1(i)}(t ; \theta)=(-1)^{i} \frac{(\theta+1)(\theta+2) \ldots(\theta+i-1)}{\theta^{i}}(t+1)^{-1 / \theta-i}
$$

as follows: First, we generate $k$ independent, uniformly distributed random variates $w_{i}, i=1,2, \ldots, k$. Then, we set $u_{1}=w_{1}$ and $w_{2}=C_{2 \mid 1}\left(u_{2} \mid w_{1}\right)$ with $c_{1}=\phi\left(u_{1} ; \theta\right)=u_{1}^{-\theta}-1, c_{2}=\phi\left(u_{1} ; \theta\right)+$ $\phi\left(u_{2} ; \theta\right)=u_{1}^{-\theta}+u_{2}^{-\theta}-2$, and $C_{2 \mid 1}\left(u_{2} \mid w_{1}\right)=\phi^{-1(1)}\left(c_{2}\right) / \phi^{-1(1)}\left(c_{1}\right)$. Therefore, we obtain $w_{2}=\left(\left(u_{1}^{-\theta}+u_{2}^{-\theta}-1\right) / u_{1}^{-\theta}\right)^{-1 / \theta-1}$, which leads to $u_{2}=\left(\left(w_{1}^{-\theta}\left(w_{2}^{-\theta /(\theta+1)}-1\right)\right)+1\right)^{-1 / \theta}$. Similarly, we set $w_{3}=C_{3 \mid 1,2}\left(u_{3} \mid u_{1}, u_{2}\right)=\phi^{-1(2)}\left(c_{3}\right) / \phi^{-1(2)}\left(c_{2}\right)=\left(\left(u_{1}^{-\theta}+u_{2}^{-\theta}+\right.\right.$ $\left.\left.u_{3}^{-\theta}-2\right) /\left(u_{1}^{-\theta}+u_{2}^{-\theta}-1\right)\right)^{-1 / \theta-2}$, which results in

$u_{3}=\left(w_{3}^{-\theta /(1+2 \theta)}\left(w_{1}^{-\theta}+w_{2}^{-\theta}-1\right)-w_{1}^{-\theta}-w_{2}^{-\theta}+2\right)^{-1 / \theta}$

As a result of using this recursion, we obtain

$$
\begin{aligned}
w_{k} & =C_{k \mid 1,2, \ldots, k-1}\left(u_{k} \mid u_{1}, u_{2}, \ldots, u_{k-1}\right) \\
& =\left(\frac{u_{1}^{-\theta}+u_{2}^{-\theta}+\cdots+u_{k}^{-\theta}-k+1}{u_{1}^{-\theta}+u_{2}^{-\theta}+\cdots+u_{k-1}^{-\theta}-k+2}\right)^{-1 / \theta-k+1}
\end{aligned}
$$

and thus,

$$
\begin{aligned}
u_{k}= & \left(\left(u_{1}^{-\theta}+u_{2}^{-\theta}+\cdots+u_{k-1}^{-\theta}-k+2\right)\right. \\
& \left.\times\left(w_{k}^{\theta /(\theta(1-k)-1)}-1\right)+1\right)^{-1 / \theta} .
\end{aligned}
$$

The generation of $k$ random variates from the $k$-dimensional Gumbel copula also starts with the sampling of $k$ independent, uniformly distributed random variates $w_{i}, i=1,2, \ldots, k$. Then, we set $u_{1}=w_{1}$ and $w_{2}=C_{2 \mid 1}\left(u_{2} \mid w_{1}\right)=\phi^{-1(1)}\left(c_{2}\right) / \phi^{-1(1)}\left(c_{1}\right)$ with $c_{1}=\phi\left(u_{1} ; \theta\right)=\left(-\log \left(u_{1}\right)\right)^{\theta}$ and $c_{2}=\phi\left(u_{1} ; \theta\right)+$ $\phi\left(u_{2} ; \theta\right)=\left(-\log \left(u_{1}\right)\right)^{\theta}+\left(-\log \left(u_{2}\right)\right)^{\theta}$, and solve the resulting equation for $u_{2}$. We obtain $u_{3}, u_{4}, \ldots, u_{k}$ in a similar manner by setting $w_{i}=C_{i \mid 1,2, \ldots, i-1}\left(u_{i} \mid u_{1}, u_{2}, \ldots, u_{i-1}\right)=\phi^{-1(i-1)}$ $\left(c_{i} ; \theta\right) / \phi^{-1(i-1)}\left(c_{i-1}\right)$ and solving this equation for $u_{i}, i=3,4$, $\ldots, k$. Thus, unlike the Clayton copula, there is no recursive formula for the inverse of the generator function of the $k$ dimensional Gumbel copula. Nevertheless, we can easily obtain $u_{1}, u_{2}, \ldots, u_{k}$ via the use of numerical search procedures.

Another way of sampling a $k$-dimensional Gumbel copula is to use the Marshall and Olkin generation method [107] with the $(1 / \theta)$-stable random variable $\gamma$ and the Laplace transform $\Theta(\gamma)=$ $\exp \left(-\gamma^{1 / \theta}\right)^{4}$. Specifically, we first generate a random variable $\gamma$ from the Stable $(1,0,0)$ distribution with parameter $1 / \theta$. We do this by sampling a uniform random variable $r$ on the interval $(-\pi / 2, \pi / 2)$ and an exponential random variable $\xi$ with mean 1 , and setting

$$
\gamma=\frac{\sin (r / \theta)}{(\cos (r))^{\theta}}\left[\frac{\cos ((1-1 / \theta) r)}{\xi}\right]^{(1-1 / \theta) /(1 / \theta)} .
$$

Then, independent of the previous step, we generate $k$ independent uniform random variates $w_{i}, i=1,2, \ldots, k$ and apply the transformation $u_{i}=\Theta\left(-\log \left(w_{i}\right) / \gamma\right)$ for $i=1,2, \ldots, k$. Similarly, the Marshall and Olkin generation method can be used for sampling from a $k$-dimensional Clayton copula with $\gamma$ defined as a gamma random variable having shape parameter 1 and scale parameter $1 / \theta$ and the Laplace transform $\Theta(\gamma)$ defined by $(1+\gamma)^{-1 / \theta}[108]$.

\subsection{Sampling from the $\mathcal{C}$-vine and $\mathcal{D}$-vine specifications}

The sampling of a random vector with a copula-vine specification starts with the generation of $k$ independent uniform random variates $w_{i}, i=1,2, \ldots, k$; proceeds by traversing the regular vine of interest in a specific order; and applying successive inversions of the conditional distributions derived from the two-dimensional copulas of each edge. Specifically, the sampling algorithm associated with a $\mathcal{C}$-vine specification is based on the graphical representation of the $k$-dimensional distribution with 
(conditional) tail dependencies assigned to the following edges of the vine:

$\begin{array}{lllll}1,2 & 1,3 & 1,4 & \cdots & 1, k \\ & 2,3 \mid 1 & 2,4 \mid 1 & \ldots & 2, k \mid 1 \\ & & 3,4 \mid 1,2 & \ldots & 3, k \mid 1,2 \\ & & \ddots & \vdots \\ & & & k-1, k \mid 1,2, \ldots, k-2\end{array}$

In particular, " $1, k$ " refers to the edge between the (unconditional) random variables $X_{1}$ and $X_{k}$, while " $k-1, k \mid 1,2, \ldots, k-2$ " represents the edge associated with the conditional random variables $X_{k-1} \mid X_{1}, X_{2}, \ldots, X_{k-2}$ and $X_{k} \mid X_{1}, X_{2}, \ldots, X_{k-2}$. Thus, we sample the uniform random vector $\left(U_{1}, U_{2}, \ldots, U_{k}\right)^{\prime}$ with a $k$ dimensional distribution function represented by a $\mathcal{C}$-vine as follows:

$$
\begin{aligned}
& u_{1} \leftarrow w_{1} . \\
& u_{2} \leftarrow C_{2 \mid 1}^{-1}\left(w_{2} \mid u_{1}\right) . \\
& u_{3} \leftarrow C_{3 \mid 1}^{-1}\left(C_{3 \mid 2: 1}^{-1}\left(w_{3} \mid u_{2}\right) \mid u_{1}\right) . \\
& u_{4} \leftarrow C_{4 \mid 1}^{-1}\left(C_{4 \mid 2: 1}^{-1}\left(C_{4 \mid 3: 1,2}^{-1}\left(w_{4} \mid u_{3}\right) \mid u_{2}\right) \mid u_{1}\right) . \\
& \vdots \\
& u_{k} \leftarrow C_{k \mid 1}^{-1}\left(C _ { k | 2 : 1 } ^ { - 1 } \left(C _ { k | 3 : 1 , 2 } ^ { - 1 } \left(\cdots \left(C_{k \mid k-1: 1,2, \ldots, k-2}^{-1}\right.\right.\right.\right. \\
&\left.\left.\left.\left.\left.\quad\left(w_{k} \mid u_{k-1}\right) \mid u_{k-2}\right) \cdots\right) \mid u_{3}\right) \mid u_{2}\right) \mid u_{1}\right) .
\end{aligned}
$$

In this sampling algorithm, $C_{i \mid j}$ is the conditional distribution associated with the bivariate copula between random variables $U_{i}$ and $U_{j}$ (i.e., $\left.C_{i \mid j}\left(u_{i} \mid u_{j}\right)=\partial C_{2}\left(u_{i}, u_{j}\right) / \partial u_{j}\right)$, while $C_{i \mid j: 1,2, \ldots, j-1}$ is the conditional distribution associated with the conditional copula $C_{i, j \mid 1,2, \ldots, j-1}$ between the random variables $U_{i} \mid U_{1}, U_{2}, \ldots, U_{j-1}$ and $U_{j} \mid U_{1}, U_{2}, \ldots, U_{j-1}$; i.e.,

$C_{i \mid j: 1,2, \ldots, j-1}\left(u_{i} \mid u_{j}\right)=\frac{\partial C_{2}\left(u_{i}, u_{j} \mid u_{1}, u_{2}, \ldots, u_{j-1}\right)}{\partial u_{j}}$.

The uniform random variates $w_{1}, w_{2}, \ldots, w_{k}$ are generated independently and $u_{1}, u_{2}, \ldots, u_{k}$ are obtained by applying successive inverse cdfs. Furthermore, the first four rows of this sampling algorithm describe the generation of random variates $u_{1}, u_{2}, u_{3}$, and $u_{4}$ from the four-dimensional $\mathcal{C}$-vine illustrated in Fig. 4 .

When the joint distribution function of the random variables $U_{1}, U_{2}, \ldots, U_{k}$ is specified by a $\mathcal{D}$-vine, which is illustrated in Fig. 3 for a four-dimensional random vector, the graphical representation is obtained in terms of the dependence measures associated with the following edges of the vine:

\begin{tabular}{lllll}
\hline 1, & $1,3 \mid 2$ & $1,4 \mid 2,3$ & $\ldots$ & $1, k \mid 2, \ldots, k-1$ \\
2 & & & & \\
& 2,3 & $2,4 \mid 3$ & $\cdots$ & $2, k \mid 3, \ldots, k-1$ \\
& & 3,4 & $\cdots$ & $3, k \mid 4, \ldots, k-1$ \\
& & $\ddots$ & $\vdots$ \\
& & & $k-2, k \mid k-1$ \\
& & & & \\
& & & & $k-1, k$ \\
\hline
\end{tabular}

Therefore, we first generate $k$ independent uniform random variates $w_{1}, w_{2}, \ldots, w_{k}$ and then sample the uniform random vector $\left(U_{1}, U_{2}, \ldots, U_{k}\right)^{\prime}$ represented with the $\mathcal{D}$-vine by calculating

$$
\begin{aligned}
& u_{1} \leftarrow w_{1}, \\
& u_{2} \leftarrow C_{2 \mid 1}^{-1}\left(w_{2} \mid u_{1}\right), \\
& u_{3} \leftarrow C_{3 \mid 2}^{-1}\left(C_{3 \mid 1,2}^{-1}\left(w_{3} \mid C_{1 \mid 2}\left(u_{1} \mid u_{2}\right)\right) \mid u_{2}\right),
\end{aligned}
$$

$$
\begin{aligned}
& u_{4} \\
& \leftarrow C_{4 \mid 3}^{-1}\left(C_{4 \mid 2,3}^{-1}\left(C_{4 \mid 1,2,3}^{-1}\left(w_{4} \mid C_{1 \mid 2,3}\left(u_{1} \mid u_{2}, u_{3}\right)\right) \mid C_{2 \mid 3}\left(u_{2} \mid u_{3}\right)\right) \mid u_{3}\right), \\
& \vdots \\
& \begin{aligned}
u_{k} \leftarrow & C_{k \mid k-1}^{-1}\left(C _ { k | k - 2 , k - 1 } ^ { - 1 } \left(\cdots C _ { k | 1 , 2 , \ldots , k - 1 } ^ { - 1 } \left(w_{k} \mid C_{1 \mid 2,3, \ldots, k-1}\right.\right.\right. \\
& \left.\left.\left.\quad\left(u_{1} \mid u_{2}, u_{3}, \ldots, u_{k-1}\right)\right) \cdots \mid C_{k-2 \mid k-1}\left(u_{k-2} \mid u_{k-1}\right)\right) \mid u_{k-1}\right) .
\end{aligned}
\end{aligned}
$$

Thus, the sampling procedure for $\mathcal{D}$-vine uses both conditional distributions and inverse conditional distributions. The first four rows of this sampling procedure further describe the sampling of a uniform random vector from the four-dimensional $\mathcal{D}$-vine in Fig. 3.

\section{Conclusion}

As large-scale stochastic simulation becomes a tool that is used routinely for the design and analysis of complex systems, it is important to develop multivariate input models that are flexible enough to capture the joint distributional properties of the system inputs. A close look at the existing literature shows that the development of a large number of multivariate input models builds on the use of the normal copula for dependence modeling. However, the normal copula fails to represent the dependence structures with tail dependencies that arise in the context of extreme events.

In this survey, we reviewed the copula-based input models for stochastic simulations with dependent inputs by focusing on the tail dependence. First, we reviewed the bivariate copula models along with their tail dependence properties and then extended our discussion to the multivariate copula models with three or more component random variables. Finally, we discussed how to fit these copula models to the available historical data sets, and how to generate random vectors from the resulting joint distributions.

The case of bivariate copulas with tail-dependence power has been well studied, but these do not readily extend to multiple dimensions. Recently, several multivariate parametric copulas have been introduced, but the study of their tail-dependence properties is still in its infancy. We believe that the study of the taildependence characteristics of the existing multivariate copulas as well as the development of new multivariate copulas that have the ability to capture a wide variety of asymmetric dependence structures are the promising research areas to enhance the field of multivariate input modeling for stochastic simulations.

\section{References}

[1] S. Vincent, Input data analysis, in: J. Banks (Ed.), Handbook of Simulation, John Wiley \& Sons, New York, 1998, pp. 55-91.

[2] A.M. Law, Simulation Modeling and Analysis, fourth ed., McGraw-Hill, New York, 2007.

[3] M.E. Kuhl, J.S. Ivy, E.K. Lada, N.M. Steiger, M.A. Wagner, J.R. Wilson, Univariate input models for stochastic simulation, Journal of Simulation 4 (2010)81-97.

[4] S.H. Xu, Structural analysis of a queuing system with multi-classes of correlated arrivals and blocking, Operations Research 47 (1999) 264-276.

[5] M. Livny, B. Melamed, A.K. Tsiolis, The impact of autocorrelation on queuing systems, Management Science 39 (1993) 322-339.

[6] P.P. Ware, T.W. Page, B.L. Nelson, Automatic modeling of file system workloads using two-level arrival processes, ACM Transactions on Modeling and Computer Simulation 8 (1998) 305-330.

[7] B. Biller, I. Civelek, A. Scheller-Wolf, Impact of dependence on singleserver queueing systems, Tepper Working Paper 2010-E18, Carnegie Mellon University, Pittsburgh, PA, 2010.

[8] A.A.B. Pritsker, D.L. Martin, J.S. Reust, M.A.F. Wagner, J.R. Wilson, M.E. Kuhl, J.P. Roberts, O.P. Daily, A.M. Harper, E.B. Edwards, L. Bennett, J.F. Burdick, M.D. Allen, Organ transplantation policy evaluation, in: C. Alexopoulos, K. Kang, W. R. Lilegdon, D. Goldsman (Eds.), Proceedings of the 1995 Winter Simulation Conference, 1995, pp. 1314-1323.

[9] S. Ghosh, M.S. Squillante, Analysis and control of web server queues, Computer Communications 27 (2004) 1771-1785.

[10] A.N. Avramidis, A. Deslauriers, P. L'Ecuyer, Modeling daily arrivals to a telephone call center, Management Science 50 (2004) 896-908. 
[11] P. Kouvelis, P. Su, The Structure of Global Supply Chains, in: Research Monograph for Book Series on Foundations and Trends ${ }^{@}$ in Technology, Information, and Operations Management, NOW, Boston, Delft, 2007.

[12] B. Biller, S. Ghosh, Multivariate input processes, in: B.L. Nelson, S.G. Henderson (Eds.), Handbooks in Operations Research and Management Science: Simulation, Elsevier Science, Amsterdam, 2006, pp. 123-153.

[13] A.J. Patton, Modeling asymmetric exchange rate dependence, International Economic Review 47 (2005) 527-556.

[14] C.J. Corbett, K. Rajaram, A generalization of the inventory pooling effect to nonnormal dependent demand, Manufacturing and Service Operations Management 8 (2006) 351-358.

[15] S.M. Wagner, C. Bode, P. Koziol, Supplier default dependencies: empirical evidence from the automotive industry, European Journal of Operational Research 199 (2009) 150-161.

[16] B.M. Tehrani, S.H. Xu, S. Kumara, H. Li, A single-period analysis of a two-echelon inventory system with dependent supply uncertainty, Transportation Research Part B: Methodological 45 (2011) 1128-1151.

[17] T.A. Craney, N. White, Distribution selection with no data using VBA and excel, Quality Engineering 16 (2004) 643-656.

[18] R.B. Nelsen, An Introduction to Copulas, second ed., Springer-Verlag, New York, 2006.

[19] K. Jong-Dev, Measures of dependence, in: P.R. Krishnaiah, P.K. Sen (Eds.), Handbook of Statistics, Elsevier Science, North-Holland, Amsterdam, 1984, pp. 79-88.

[20] G. Yule, M.G. Kendall, An Introduction to the Theory of Statistics, Griffin, London, 1965.

[21] W. Whitt, Bivariate distributions with given marginals, The Annals of Statistics 4 (1976) 1280-1289.

[22] H. Hotelling, M.R. Pabst, Rank correlation and tests of significance involving no assumption of normality, The Annals of Mathematical Statistics 7 (1936) 29-43.

[23] P. Embrechts, A. McNeil, D. Straumann, Correlation and dependence in risk management: properties and pitfalls, in: M.A.H. Dempster (Ed.), Risk Management: Value at Risk and Beyond, Cambridge University Press, Cambridge, UK, 2002, pp. 176-223.

[24] W. Hoeffding, Masstabinvariante korrelations theorie, Schrijtfen des Mathematischen Instituts und des Instituts fr Angewandte Mathematik det Universitt Berlin 5 (1940) 179-233.

[25] P.J. Danaher, M.S. Smith, Modeling multivariate distributions using copulas: applications in marketing, Marketing Science 30 (2011) 4-21.

[26] H. Joe, Multivariate Models and Dependence Concepts, Chapman and Hall, London, 1997.

[27] B. Biller, Copula-based multivariate input models for stochastic simulation, Operations Research 57 (2009) 878-892.

[28] R.D. Cook, M.E. Johnson, A family of distributions for modeling nonelliptically symmetric multivariate data, Journal of the Royal Statistical Society. Series B 43 (1981) 210-218.

[29] R.D. Cook, M.E. Johnson, Generalized Burr-Pareto-logistic distributions with applications to an uranium exploration data set, Technometrics 28 (1986) 123-131.

[30] Y.L. Tong, The Multivariate Normal Distribution, Springer-Verlag, New Jersey, 1990.

[31] N.L. Johnson, Bivariate distributions based on simple translation systems, Biometrika 36 (1949) 297-304.

[32] M.C. Cario, B.L. Nelson, Modeling and generating random vectors with arbitrary marginal distributions and correlation matrix, Working Paper, Department of Industrial Engineering and Management Sciences, Northwestern University, Evanston, IL, 1997.

[33] I. Fortin, C. Kuzmics, Tail-dependence in stock-return pairs, International Journal of Intelligent Systems in Accounting, Finance and Management 11 (2002) 89-107.

[34] A. Ang, J. Chen, Asymmetric correlations of equity portfolios, Journal of Financial Economics 63 (2002) 443-494.

[35] A. Ang, G. Bekaert, International asset allocation with regime shifts, Review of Financial Studies 15 (2002) 1137-1187.

[36] K.-H. Bae, G.A. Karolyi, R.M. Stulz, A new approach to measuring financial contagion, Review of Financial Studies 16 (2003) 717-764.

[37] M.-H. Zhang, Modeling total tail dependence along diagonals, Insurance: Mathematics and Economics 42 (2008) 73-80.

[38] Y. Sun, X. Wu, A nonparametric study of dependence between S \& P 500 index and market volatility index (VIX), Working Paper, 2009

[39] U. Cherubini, E. Luciano, Value-at-risk trade-off and capital allocation with copulas, Economic Notes 30 (2001) 235-256.

[40] P. Embrechts, F. Lindskog, A. McNeil, Modeling dependence with copulas and applications to risk management, in: S.T. Rachev (Ed.), Handbook of Heavy Tailed Distributions in Finance, Elsevier, North-Holland, 2003.

[41] P. Embrechts, A. Höing, Extreme VaR scenarios in higher dimensions, Extremes 9 (2006) 177-192.

[42] J.V. Rosenberg, Nonparametric pricing of multivariate contingent claims, Journal of Derivatives 10 (2003) 9-26.

[43] M.N. Bennett, J.E. Kennedy, Quanto pricing with copulas, Journal of Derivatives 12 (2004) 26-45.

[44] U. Cherubini, E. Luciano, W. Vecchiato, Copula Methods in Finance, John Wiley \& Sons, Chichester, UK, 2004.

[45] R.W.J. van den Goorbergh, C. Genest, B.J.M. Werker, Multivariate option pricing using dynamic copula models, Insurance: Mathematics and Economics 37 (2005) 101-114.
[46] M. Salmon, C. Schleicher, Pricing multivariate currency options with copulas, in: J. Rank (Ed.), Copulas: From Theory to Application in Finance, Risk Books, London, 2006.

[47] D.X. Li, On default correlation: a copula function approach, Journal of Fixed Income 9 (2000) 43-54.

[48] K. Giesecke, Correlated default with incomplete information, Journal of Banking and Finance 28 (2004) 1521-1545.

[49] A.J. Patton, On the out-of-sample importance of skewness and asymmetric dependence for asset allocation, Journal of Financial Econometrics 2 (2004) 130-168.

[50] R. Garcia, G. Tsafack, Dependence structure and extreme co-movements in international equity and bond markets, Journal of Banking and Finance 35 (2011) 1954-1970.

[51] A.J. Patton, Copula-based models for financial time series, in: Handbook of Financial Time Series, Springer-Verlag, 2009, pp. 767-781 (Chapter V-3).

[52] R.T. Clemen, T. Reilly, Correlations and copulas for decision and risk analysis, Management Science 45 (1999) 208-224.

[53] M.N. Jouini, R.T. Clemen, Copula models for aggregating expert opinions, Operations Research 44 (1996) 444-457.

[54] D. Vedenov, Application of copulas to estimation of joint crop yield distributions, in: Proceedings of the American Agricultural Economics Association Annual Meeting, Orlando, FL, 2008.

[55] J. Palomo, D. Rios Insua, F. Ruggeri, Modeling external risks in project management, Risk Analysis 27 (2007) 961-978.

[56] W. Yi, V.W. Bier, An application of copulas to accident precursor analysis, Management Science 44 (1998) 257-270.

[57] A. Meel, W.D. Seider, Real-time risk analysis of safety systems, Computers and Chemical Engineering 32 (2008) 827-840

[58] C.R. Bhat, N. Eluru, A copula-based approach to accommodate residential selfselection effects in travel behavior modeling, Transportation Research Part B: Methodological 43 (2009) 749-765.

[59] K. Takahasi, Note on the multivariate Burr's distribution, Annals of the Institute of Statistical Mathematics 17 (1965) 257-260.

[60] N.L. Johnson, S. Kotz, Distributions in Statistics: Continuous Multivariate Distributions, John Wiley \& Sons, New York, 1972.

[61] S.P. Satterthwaite, T.P. Hutchinson, A generalization of Gumbel's bivariate logistic distribution, Metrika 25 (1978) 163-170.

[62] R.L. Plackett, A class of bivariate distributions, Journal of American Statistical Association 60 (1965) 516-522.

[63] D. Morgenstern, Einfache beispiele zweidimensionaler verteilungen, Mitteilingsblatt fr Mathematishe Statistik 8 (1956) 234-235.

[64] N.L. Johnson, S. Kotz, On some generalized Farlie-Gumbel-Morgenstern distributions, Communications in Statistics 4 (1975) 415-427.

[65] M.E. Johnson, Multivariate Statistical Simulation, Wiley, New York, 1987.

[66] C. Genest, R.J. MacKay, The joy of copulas: bivariate distributions with uniform marginals, The American Statistician (American Statistical Association) 40 (1986) 280-283.

[67] B. Schweizer, Thirty years of copulas, in: G. Dall'Aglio, S. Kotz, G. Salinetti (Eds.), Advances in Probability Distributions with Given Marginals, Kluwer, Dordrecht, Netherlands, 1991, pp. 13-50.

[68] P. Trivedi, D. Zimmer, Copula modeling: an introduction for practitioners, Foundations and Trends in Econometrics 1 (2005) 1-111.

[69] N.L. Johnson, Systems of frequency curves generated by methods of translation, Biometrika 36 (1949) 149-176.

[70] D. Kelker, Distribution theory of spherical distributions and location-scale parameter generalization, Sankhyā 32 (1970) 419-430.

[71] K. Fang, S. Kotz, K.W. Ng, Symmetric Multivariate and Related Distributions, Chapman \& Hall, London, 1987.

[72] G. Kimeldorf, A.R. Sampson, Uniform representations of bivariate distributions, Communications in Statistics 4 (1975) 617-627.

[73] E.J. Gumbel, Distributions des valeurs extrêmes en plusiers dimensions, Publications de LiInstitute de Statistique 9 (1960) 171-173.

[74] A.A. Balkema, S.I. Resnick, Max-infinite divisibility, Journal of Applied Probability 14 (1977) 309-319.

[75] D. MacKenzie, End-of-the-world trade, in: London Review of Books, Vol. 30, 2008, pp. 24-26.

[76] F. Salmon, Recipe for disaster: the formula that killed Wall Street, 2009. Wired 02/23/2009.

[77] A. Lipton, A. Rennie, Credit Correlation: Life After Copulas, World Scientific Publishing Co., Hackensack, NJ, 2007.

[78] C. Donnelly, P. Embrechts, The devil in the tails: actuarial mathematics and the subprime mortgage crisis, ASTIN Bulletin 40 (2010) 1-33.

[79] D. Brigo, A. Pallavicini, R. Torresetti, Credit Models and the Crisis: A Journey into CDOs, Copulas, Correlations, and Dynamic Models, in: The Wiley Finance Series, 2010.

[80] E. Gomeza, M.A. Gomez-Viilegasa, J.M. Marina, A multivariate generalization of the power exponential family of distributions, Communications in Statistics: Theory and Methods 27 (1998) 589-600.

[81] Z.M. Landsman, E.A. Valdez, Tail conditional expectations for elliptical distributions, Technical Report N 02-04, 2002.

[82] B.V.M. Mendes, O. Arslan, Multivariate skew distributions based on the GT copula, Brazilian Review of Econometrics 26 (2006) 235-255.

[83] C.H. Kimberling, A probabilistic interpretation of complete monotonicity, Aequationes Mathematicae 10 (1974) 152-164.

[84] J. Galambos, Order statistics of samples from multivariate distributions, Journal of American Statistical Association 70 (1975) 674-680. 
[85] R. Cooke, Markov and entropy properties of tree and vines-dependent variables, in: Proceedings of the ASA Section on Bayesian Statistical Science, American Statistical Association, Alexanria, VA, 1997, pp. 166-175.

[86] T. Bedford, R.M. Cooke, Probability density decomposition for conditionally dependent random variables modeled by vines, Annals of Mathematics and Artificial Intelligence 32 (2001) 245-268.

[87] T. Bedford, R.M. Cooke, Vines-a new graphical model for dependent random variables, The Annals of Statistics 30 (2002) 1031-1068.

[88] D. Kurowicka, R. Cooke, A parameterization of positive definite matrices in terms of partial correlation vines, Linear Algebra and its Applications 372 (2003) 225-251.

[89] K. Aas, C. Czado, A. Frigessi, H. Bakken, Pair-copula constructions of multiple dependence, Insurance: Mathematics and Economics 44 (2009) 182-198.

[90] D. Kurowicka, R. Cooke, Uncertainty Analysis with High Dimensional Dependence Modeling, in: Wiley Series in Probability and Statistics, 2006.

[91] P.A. Maugis, D. Guegan, New prospects on vines, Insurance Markets and Companies: Analysis and Actuarial Computations 1 (2010) 15-22.

[92] H. Joe, L. Haijun, A.K. Nikoloulopoulos, Tail dependence functions and vine copulas, Journal of Multivariate Analysis 101 (2010) 252-270.

[93] R.J. Serfling, Approximation Theorems of Mathematical Statistics, Wiley, New York, 1980.

[94] P. Billingsley, Statistical Inference for Markov Processes, University of Chicago Press, Chicago, 1961.

[95] G. Kim, M.J. Silvapulle, P. Silvapulle, Comparison of semi-parametric and parametric methods for estimating copulas, Communications in Statistics: Simulation and Computation 51 (2007) 2836-2850.

[96] L. Dalla Valle, Bayesian copulae distributions, with application to operational risk management, Methodology and Computing in Applied Probability 11 (2009) 95-115.
[97] P.E. Rossi, G.M. Allenby, R. McCulloch, Bayesian Statistics and Marketing, in: Wiley Series in Probability and Statistics, 2006.

[98] W.R. Gilks, S. Richardson, D.J. Spiegelhalter, Markov Chain Monte Carlo in Practice, Chapman and Hall, London, UK, 1996.

[99] C. Czado, A. Min, Bayesian inference for $\mathcal{D}$-vines: estimation and model selection, in: D. Kurowicka, H. Joe (Eds.), Dependence Modeling: Vine Copula Handbook, World Scientific Publishing Co., Singapore, 2011, pp. 249-264.

[100] D. Berg, Copula goodness-of-fit testing: an overview and power comparison, The European Journal of Finance 15 (2009) 675-701.

[101] C. Genest, B. Rémillard, D. Beaudoin, Goodness-of-fit tests for copulas: a review and a power study, Insurance: Mathematics and Economics 44 (2009) 199-213.

[102] P. Deheuvels, La fonction de dépendance empirique et ses propriétés: Un test non paramétrique d'imdépendance, Académie Royale de Belgique: Bulletin de la Classe des Sciences, 5e Série 65 (1979) 274-292.

[103] C. Genest, B. Rémillard, Validity of parametric bootstrap for goodness-offit testing in semiparametric models, Annals of Henri Poincaré 44 (2008) 1096-1127.

[104] M. Rosenblatt, Remarks on a multivariate transformation, The Annals of Mathematical Statistics 23 (1952) 470-472.

[105] G.N.F. Weiss, Are Copula-GoF tests of any practical use? empirical evidence for stocks, commodities, and FX futures, The Quarterly Review of Economics and Finance 51 (2011) 173-188.

[106] E.M. Scheuer, D.S. Stoller, On the generation of normal random vectors, Technometrics 4 (1962) 278-281.

[107] A.W. Marshall, I. Olkin, Families of multivariate distributions, Journal of American Statistical Association 83 (1988) 834-841.

[108] E.W. Frees, E. Valdez, Understanding relationships using copulas, North American Actuarial Journal 2 (1998) 1-25. 